\title{
Synthesis of Cresols and Xylenols from Benzene and Methanol
}

H.W. Prengle, Jr., F.A. Bricout, and S. Alam

University of Houston

Houston, Texas

NREL Technical Monitor: R. Gerald Nix

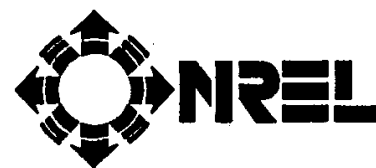

National Renewable Energy Laboratory

(formerly the Solar Energy Research Institute)

1617 Cole Boulevard

Golden, Colorado 80401-3393

A Division of Midwest Research Institute Operated for the U.S. Department of Energy under Contract No. DE-AC02-83CH10093

Prepared under Subcontract No. XX-7-07028-1

April 1992 
This report describes subcontracted research. The report is unreviewed and expresses only the opinions of the author[s]. It has been prepared for reproduction from the best available copy.

\title{
NOTICE
}

This report was prepared as an account of work sponsored by an agency of the United States government. Neither the United States government nor any agency thereof, nor any of their employees, makes any warranty, express or implied, or assumes any legal liability or responsibility for the accuracy, completeness, or usefulness of any information, apparatus, product, or process disclosed, or represents that its use would not infringe privately owned rights. Reference herein to any specific commercial product, process, or service by trade name, trademark. manufacturer, or otherwise does not necessarily constitute or imply its endorsement, recommendation, or favoring by the United States government or any agency thereof. The views and opinions of authors expressed herein do not necessarily state or reflect those of the United States government or any agency thereof.

\author{
Printed in the United States of America \\ Available from: \\ National Technical Information Service \\ U.S. Department of Commerce \\ 5285 Port Royal Road \\ Springfield, VA 22161 \\ Price: Microfiche A01 \\ Printed Copy $\mathrm{A03}$
}

Codes are used for pricing all publications. The code is determined by the number of pages in the publication. Information pertaining to the pricing codes can be found in the current issue of the following publications which are generally available in most libraries: Energy Research Abstracts (ERA); Government Reports Announcements and Index (GRA and I); Scientific and Technical Abstract Reports (STAR); and publication NTIS-PR-360 available from NTIS at the above address. 


\section{- EXECUTIVE SUMMARY -}

The objective of the Fork is to compare tro (2) processes for manufacming cresols and zplenols: a) -a conventional catalytic process, and b) -a photo-thermal cavalytic procoss, in order to detarmine the roletive process economics. The products are used primarity as chemical intermediates for manufactare of antiozidants, pesticides, polymerization inhibitors, resins, and other products. The market is approrimately 500 milion pounds per gear.

This report is the second of tro reports, presenting resules of a process eraluation for manufacturing the products by a photo-thermal catalytic process.-

At the outset, as experimental data are not jet avalable to provide a definitive design basis, a series of assumptions vere made in order to proceed vith the eraluation. As regands capacity, an arbiorary base case plant size (fresh feed) of approximately 7.06 million kghy (15.6 million lbm/g) ves chosen, and then the economics scaled to break-eren size. Calculations indicated the folloving comparative numbers:

\section{BASE CASE:}

1) Plant Size, fresh feed $=7.056 \mathrm{E} 6 \mathrm{~kg} / \mathrm{g} ;$ products $=5.192 \mathrm{E} 6 \mathrm{~kg} / \mathrm{g}$

2) Plant Capital Cost

$\$ 2.111 \mathrm{E6}$

3) Plant Operating Cost (POC)

$\$ 3.361 \mathrm{Z6}$

4) Plant Income (PI)

$\$ 7.267 \mathrm{E6}$

5) PIPOC

6) Minimem Qsoln

$168 \mathrm{kP}$

\section{BREAK-EYEN CASE:}

1) Plant Size, fresh feed $=1.652 \mathrm{E} 6 \mathrm{~kg} / 5 ;$ products $=1.215 \mathrm{R} 6 \mathrm{kglg}$

2) Plant Capital Cost

$\$ 0.8833 \mathrm{E6}$

3) Plant Operating Cost and Income

4) Minimum Qsols

$\$ 1.70126$

$40 \mathrm{kT}$

The above capital costs are for process equipment only, and do not include equipment to deliver solar energy to the top of the reactor

The eraluation indicates that for the photo-catalytic process, the break-eren capacity is only about 2496 of the base case, and about the same pereentage of the conpentional catalytic process (Report 1 ). Assuming the total capital cost rovld double, Fhich is unlikely, Fhen the solar energy equipment is included, the orerall conclusion in faror of the photo-catalytic process rould not change. 


\section{TABLE OF CONTENTS}

Executive Summary

page

ii

Introduction 1

1.00 Process Description and Design Basis 2

Figure 1. Process Flov - Reaction Section 4

Figure 2. Process Flov - Separation Section 5

2.00 Chemical Reaction/Reactor Section 6

Table 1. Stream Hasses, Compositions, Conditions 7

\& Enthalpies

Table 2. Reactor Specifications and Estimated Cost 9

3.00 Equipment Sizing and Cost 10

Table 3. Summary of Equipment Costs 10

Table 4. Heat Exchanger Summary 11

Table 5a-5c. Distillation Columns 13

Table 6. Pump Summary 16

Table 7. Storage Tank Summary 16

4.00 Estimated Capital and Operating Costs 17

Table 8. Capital Cost Summary 17

Table 9. Operating Cost Summary 18

5.00 Profitability Analysis 19

Table 10. Plant Income from Products 19

Table 11. Calculation of First-Year Break-Even Point 20

References Cited 21

APPENDIX

Physical and Thermodjnamic Properties al

Reactant and Product Prices bl

Plant Utilities \& Costs c1

Economic Indicators d1

Srmbols \& Homenclature el

(9-30-89) 


\section{- INTRODUCTION -}

This report is the second of two report concerning the manufacture of cresols and zylenols by, 1) -a conpentional catalytic process, and 2) -a solar-thermal photo-catalytic process. The tro reports then complete vill provide a preliminary basis for comparison of the relative process economics.

The purpose of this report is to present the rork accomplished on sizing and costing a solar photo-thermal catalytic process to produce the products from benzene and methanol. No process configuration, sizing, and costing vere available; therefore, a series of assumptions rere made in order to cans out the necessary calculations wobtain process details and the economics. The method for comparison of the tro processes vas to determine the "break-even" capacity for each process.

At the outset the reader should become familiar vith the sunctures of the product componnds, in order wo risualize the synthesis chemistry. In the photo-catalytic reaction, conducted Fith a large excess methanol, the benzene provides the ring surcture and the methanol provides the methyl group (by alkylation) and the hydraxyl gronp (by hydroxylation).

The reactants:

benzene
$\mathrm{C}_{6} \mathrm{H}_{6}$
$\mathrm{M}=78.113$

and the products:

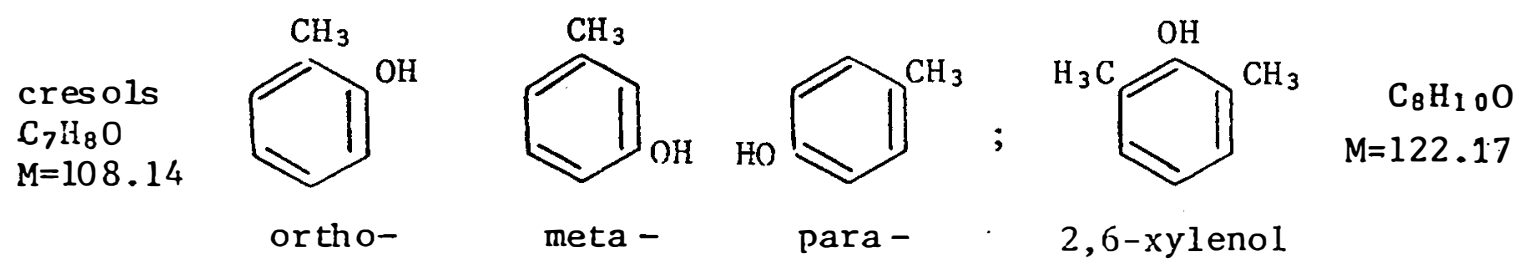

Cresol and rylenol isomers are nsed primanily as chemical intermediates. Principal applications include use for: anti- oridants, pesticides, polymerization inhibitors, resins, and other miscellaneous uses. The annual consumption of the products [2] vas presented in the first report, as vell as the U.S. price history; current reactant and product prices are presented in Appendix B . 


\subsection{PROCESS DESCRIPTION AND DESIGN BASIS}

At the outset, as experimental data are not pet available to provide a definitive design basis, the following assumptions rere made in order to proceed vith an economic evaluation of the process.

1. The orerall reaction stoichiometry for the process is,

$$
\begin{aligned}
\mathrm{C}_{6} \mathrm{H}_{6}+5 \mathrm{CH}_{3} \mathrm{OH} & ->0.500-\mathrm{C}_{7} \mathrm{H}_{8} \mathrm{O}+0.50 \mathrm{~m}, \mathrm{p}-\mathrm{C}_{7} \mathrm{H}_{8} \mathrm{O} \\
& +0.50 \mathrm{C}_{8} \mathrm{H}_{100}+3.50 \mathrm{H}_{2} \mathrm{O}+3.00 \mathrm{H}_{2}
\end{aligned}
$$

For this endothermic reaction the $\Delta \mathrm{Hr}^{0}(298 \mathrm{~K})=595.100 \mathrm{~kJ} / \mathrm{kgmole}$ benzene.

2. The Reaction Section of the plant rill operate only during sun hours"= $2500 \mathrm{~h} / \mathrm{F}$ : Fhile the Separation Section of the plant vill operate $24 \mathrm{~h} / \mathrm{d}=8400 \mathrm{~h} / \mathrm{g}$ (dorntime $=360 \mathrm{~h} / \mathrm{f})$. The solar scale-factor becomes $8400 / 2500=3.36$

\begin{tabular}{|c|c|}
\hline Fresh Feed & 7.056 million $\mathrm{kg} / \mathrm{\gamma}=15.56$ million $1 \mathrm{bm} / 7$ \\
\hline o-Cresols & 1.601 million $\mathrm{kg} / \mathrm{T}=3.530 \operatorname{million} 1 \mathrm{~b} / \mathrm{T}$ \\
\hline m.p-Cresols & on $\mathrm{kg} / \mathrm{\gamma}=3.530 \mathrm{mi}$ \\
\hline Iflenols & $\mathrm{m} \mathrm{kg} / \mathrm{g}=3.988 \mathrm{II}$ \\
\hline Hgdro & ion $1 \mathrm{~b} /$ \\
\hline
\end{tabular}

3. The fresh feed and production rates are:

4. Solar energy input, in an amount necessary to supp1y the reaction energy requirement, Fill be surficient to provide the necessary VIS-UV energy to actipate the catalgst.

5. The plant is a process unit zithin an existing chemical plant Fhere utilities and other services are arailable.

The reader is referred to Figure 1 (p 4 ) Process F1ov - Reaction Section, and Figure 2 (p6) Process Flov - Separation Section. A mass balance at key points through the process, as rell as process conditions. are shovn on the diagrams. Because the Reaction Section rould operate on a reduced number of hours/day. Fhereas the Separation Section rould operate $24 \mathrm{~h} / \mathrm{d}$, a "solar scale factor, $\mathrm{Sf}=3.36^{-}$has been included. Fhich means that on a yearly-arerage the Reaction Section rould operate $24 / 3.36=7.14 \mathrm{~h} / \mathrm{d}$.

The process nov for the Reaction Section (refer to Figure 1) proceeds as follors. Fresh feed as liquid (benzene and methanol) enters as stream $\underline{1}$ is pumped as stream $\underline{2}$ through heat exchanger $E 1$ to recoper heat from the cresol-rylenol bottoms stream (14) from the primary fractionator (PE). The feed stream, 2 flors through exchanger E2 to pick up 
additional heat and raporize before entering the reactor (REAC). The heat transferred in $E 2$ is provided by cooling the reactor effiuent stream 2. In the reactor the feed is converted to products by the overall stoichiometry shovn above. In heat exchanger E2 the reactor effluent is partially condensed and flovs as stream 6 to the primary fractionator (PF). The primary runction of PF is to remove the hydrogen as stream $\underline{8}$ the vater as stream 11 and the cresols and rylenols as the bottoms stream 14. The overhead condenser loop is the stream sequence 7.8 .9$. 10 and the reboiler loop is streams $12,13,14$. After giving up heat in $E 1$. the excess flov of stream 15 is partialiy stored in accumulator $\Delta 1$, Fhile the flov to the Separation Section is stream 17 .

The process flov for the Separation Section. Figure 2 (p5). proceeds as follows. The accumulated material in $\Lambda 1$ is pumped as stream 17 There it is divided into streams $\underline{18} \underline{20}$ and $\underline{21}$. Stream $\underline{18}$ flows through heat exchanger $E 5$ to recover heat from the cresol overhead stream (27) from the o-cresol-rylenol separation column (OCX) and exits as stream 22. Stream 20 enters heat exchanger $E 6$ vhere it recovers heat from the zylenol bottoms stream (34) from the cresol-rylenol separation column (CX) and exits as stream 23. Stream 21 flovs through heat exchanger $E 7$ to recorer heat from the cresol overhead stream (31) from $C I$ and exits as stream 24. Streams $\underline{22} \underline{23}$ and $\underline{24}$ recombine and enter the o-cresol-rylenol separation column (OCX). The main runction of OCX is to separate o-cresol as overhead stream $\underline{27}$ and $m, p$-cresol and zylenol as bottoms stream 29 . Stream $\underline{27}$ is cooled in E5 and enters the o-cresol storage tank as stream 28. The OCX bottoms stream is pumped as stream 30 vhere it enters the cresol-rylenol separation column (CX). The primary runction of $C X$ is to remove $m, p$-cresols as overhead stream 21 and rylenol as bottoms stream 34 . Stream 11 is cooled in E7 and exits to the rylenol storage tank as stream 32 . Stream 34 is cooled in E6 and enters the $m$.p-cresol storage tank as stream 32.

The major pieces of equipment are:

1-reactor, 3-fractionators, 4-accumulators, 11-heat exchangers,

7-pumps, and 5-storage tanks. 


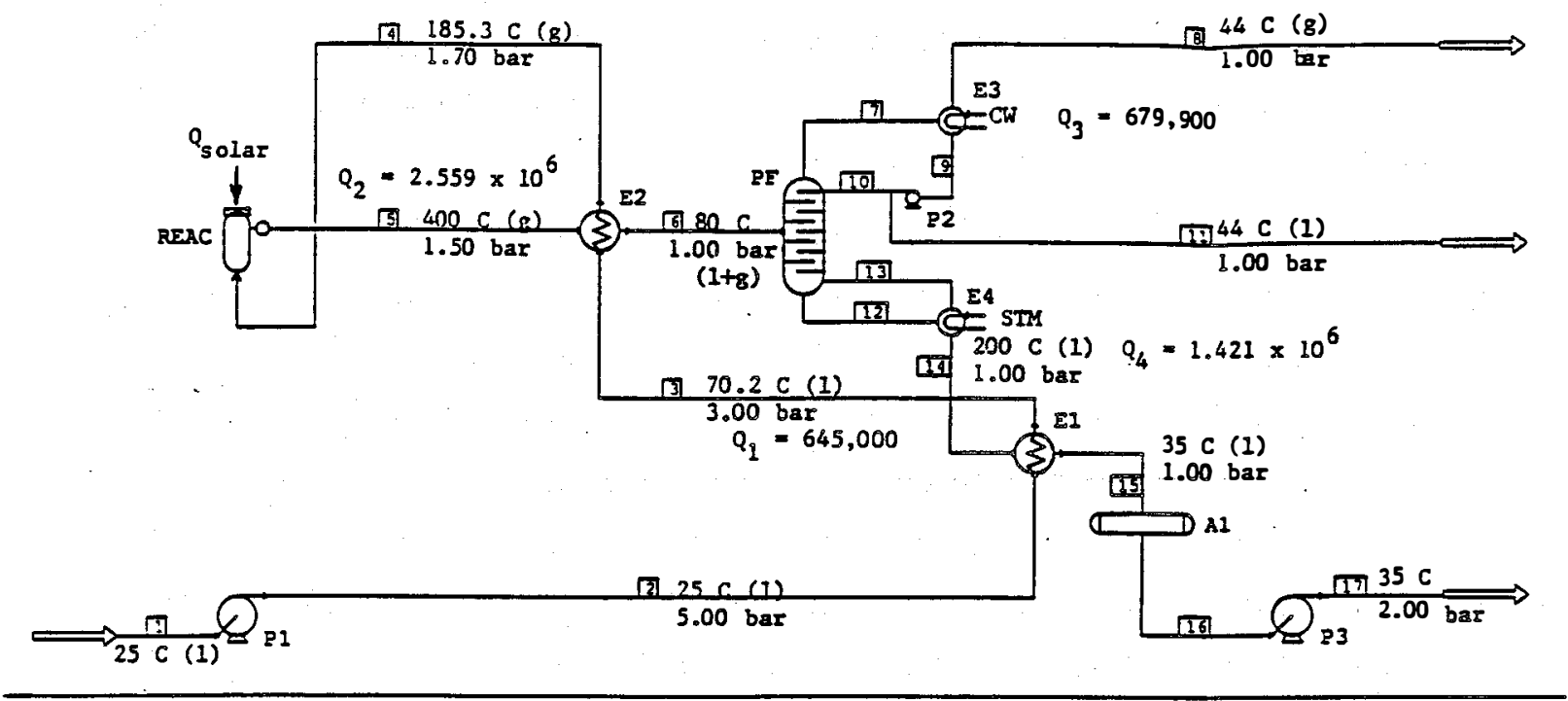

Solar Factor, $S_{f}=3.36 \quad$ streams and flow rates, $\mathrm{kg} / \mathrm{h}$

\begin{tabular}{lcccccccc} 
Component & M.H. & 1 & 5 & 14,15 & 8 & 11 & 17 \\
\hline a) BENZENE & 78.113 & 925.17 & 0.0000 & 0.0000 & 0.0000 & 0.0000 & 0.0000 \\
b) METHANOL & 32.024 & 1897.5 & 0.0000 & 0.0000 & 0.0000 & 0.0000 & 0.0000 \\
c) O-CRESOL & 108.14 & 0.0000 & 640.41 & 640.41 & 0.0000 & 0.0000 & 190.59 \\
d) M-CRESOL & 108.14 & 0.0000 & 320.20 & 320.20 & 0.0000 & 0.0000 & 95.293 \\
e) P-CRESOL & 108.14 & 0.0000 & 320.20 & 320.20 & 0.0000 & 0.0000 & 95.293 \\
f) MIXED XYLENOLS & 122.17 & 0.0000 & 723.49 & 723.49 & 0.0000 & 0.0000 & 215.31 \\
8) WATER & 18.02 & 0.0000 & 747.04 & 0.0000 & 3.2300 & 747.04 & 0.0000 \\
h) HYDROGEN & 2.016 & 0.0000 & 71.633 & 0.0000 & 32.302 & 0.0000 & 0.0000 \\
\hline
\end{tabular}

\section{Figure 1. PROCESS FLOW - REACTION SECTION}

(Note: Q-Values are $\mathrm{kJ} / \mathrm{h}$ )

$-4-$ 


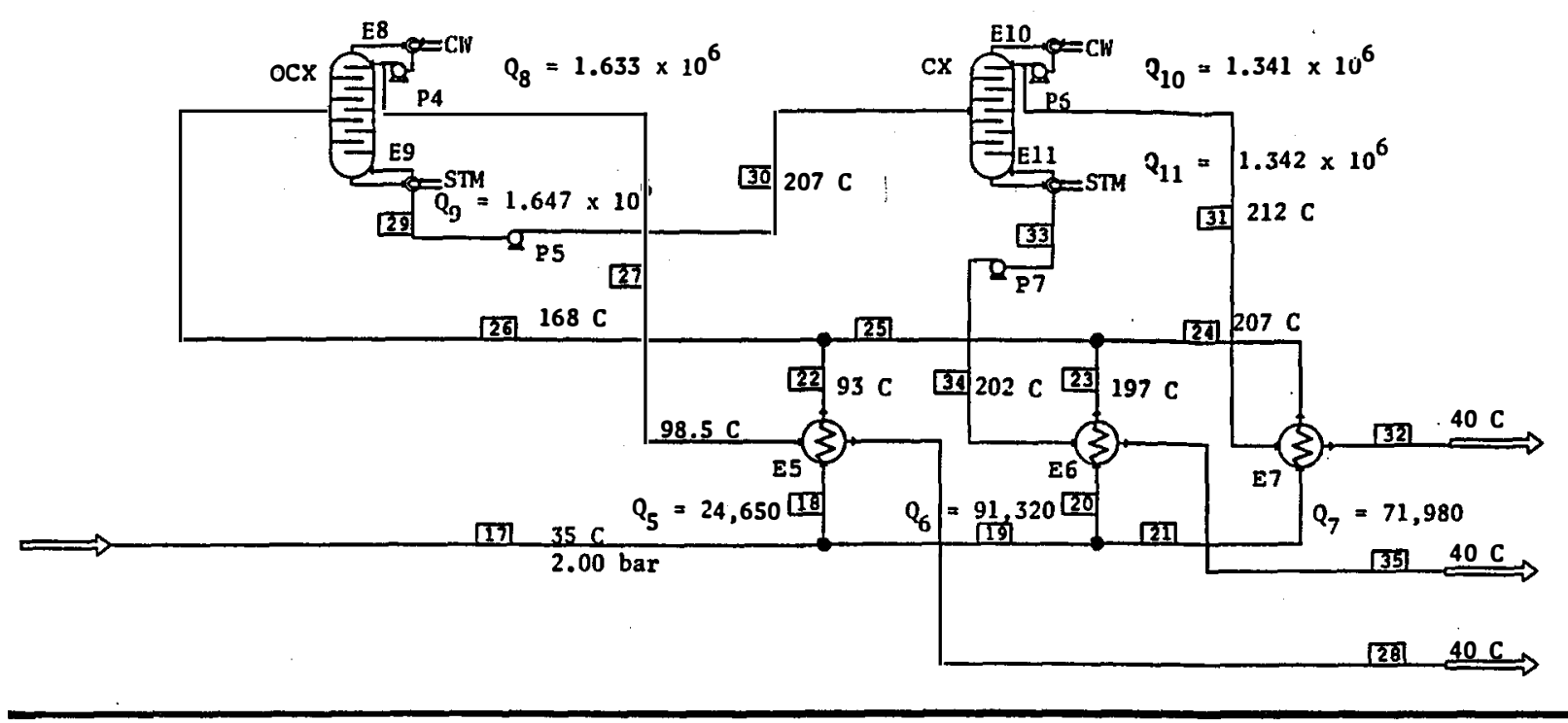

streams and flowrates, $\mathrm{kg} / \mathrm{h}$

\begin{tabular}{lllllll} 
Component & N.W. & 17,26 & 27,28 & 29,30 & 31,32 & 34,35 \\
\hline g) WATER & 18.02 & 1.1118 & 1.1118 & 0.0000 & 0.0000 & 0.0000 \\
c) O-CRESOL & 108.14 & 190.33 & 188.42 & 1.9033 & 1.9033 & 0.0000 \\
d) m-CRESOL & 108.14 & 95.300 & 0.0953 & 95.208 & 94.254 & 0.9521 \\
e) P-CRESOL & 108.14 & 95.300 & 0.1062 & 95.197 & 94.518 & 0.6768 \\
f) MIXED XYLENOLS & 122.17 & 215.32 & 0.0000 & 215.32 & 0.4306 & 214.89 \\
\hline & & & & & & \\
\hline
\end{tabular}

Figure 2. PROCESS FLOW - SEPARATION SECTION

(Note: Q-Values are kJ/h) 


\subsection{CHEMICAL REACTION/REACTOR SECTION}

The Reaction Section is the most important part of the plant as it carries out the conversion of feedstock to synthesized products. In this part of the report the details of the Reaction Section are presented.

The reader is referred to the Hov diagram. Figure 1 , vhere the stream numbers, temperatures, pressures, and heat exchanger Q-values are shovn on the diagram. Table 1 presents the stream masses. compositions, conditions, and enthalpies for key streams in the Reaction Section. It vill be noted that the solar energy input can be calculated by.

$$
\begin{aligned}
\text { Qsolar } & =m \Delta H=m 5 H 5-m 4 H s=-9.579 \mathrm{E} 6-(-10.183 \mathrm{E6}) \\
& =0.605 \mathrm{E} 6 \mathrm{~kJ} / \mathrm{h} \text { (endothermic) }=168 \mathrm{~kW}
\end{aligned}
$$

vhich assumes that vithin this total "thermodynamic" amount of energy there is surficient VIS-OV to photo-activate the catalyst. At present it is our expectation that the final amount vill not be more than 2 or 3 times the above calculated ralue. If the value turns out to be.more than the thermodynamic value, then the excess rould be used to generate steam, and a credit taken on the utility bill. A measured value of the energy requirement is yet to be determined by Vemtrorth's Research Group.

Sizing and costing calculations vere made for the shallov (h/D = 1/4) fluidized bed reactor, and are summarized in Iable 2 . The reactor size is approximately: $45^{\circ}$ i.d. I $10^{\circ}$ s to $\mathrm{s}$, vith a $2^{\circ}$ dia. quartz vindor in the top: the reactor vould be lined vith high temperature refractory cement. vhich is typical of commercial nuid-bed catalytic reactors. 


\section{Table 1. Stream Masses, Compositions, Conditions \& Enthalpies}

Datum state: enthalpies and entropies $=0$, for the chemical elements in their naturally occuring physical

state at $298.16 \mathrm{~K}$. Liquid phase mixing enthalpies are assumed small cf. formation enthalpies.

Components: benzene (a), methanol (b), o-cresol (c), m-cresol (d), p cresol (e), mixed xylenols ( $f$ ), water ( $g$ ), and hydrogen $(h)$.

\begin{tabular}{|c|c|c|c|c|c|c|c|}
\hline \multirow[b]{2}{*}{ Stream } & \multirow{2}{*}{$\begin{array}{r}\mathrm{kg} / \mathrm{h} \\
\mathrm{kgmols} / \mathrm{h} \\
\end{array}$} & & \multicolumn{2}{|c|}{ composition } & \multirow{2}{*}{$\begin{array}{c}\text { conditions } \\
\text { T.P }\end{array}$} & \multicolumn{2}{|c|}{ enthalpies } \\
\hline & & & $x$ & $y$ & & $\mathrm{~kJ} / \mathrm{kgmol}$ & $\mathrm{kJ} / \mathrm{h}$ \\
\hline$\underline{2}(\mathrm{~L})$ & $\begin{array}{l}2822.1 \\
71.050\end{array}$ & $\begin{array}{l}\mathrm{a} \\
\mathrm{b}\end{array}$ & $\begin{array}{l}0.1667 \\
0.8333\end{array}$ & & $\begin{array}{c}25 \mathrm{C}=298 \mathrm{~K} \\
5.00 \text { bars }\end{array}$ & $\mathrm{H}_{2}=-188,407$ & $\mathrm{~m}_{2} \mathrm{H}_{2}=-13.386 \mathrm{E} 6$ \\
\hline$\underline{4}(G)$ & $(")$ & $\mathrm{a}$ & & $\begin{array}{l}0.1667 \\
0.8333\end{array}$ & $\begin{array}{c}185.3 \mathrm{C}=458.3 \mathrm{~K} \\
1.70 \text { bars }\end{array}$ & $\mathrm{H}_{4}=-143,320$ & $\mathrm{~m}_{4} \mathrm{H}_{4}=-10.183 \mathrm{E} 6$ \\
\hline
\end{tabular}


Table 1. (continued)

\begin{tabular}{|c|c|c|c|c|c|c|c|}
\hline Stream & $\begin{array}{c}\mathrm{kg} / \mathrm{h} \\
\mathrm{kgmols} / \mathrm{h}\end{array}$ & & \multicolumn{2}{|c|}{ composition } & $\begin{array}{c}\text { conditions } \\
\mathrm{T}, \mathrm{P}\end{array}$ & \multicolumn{2}{|c|}{ enthalpies } \\
\hline$\underline{6}(\mathrm{~L}+\mathrm{G})$ & $(" \prime)$ & $\begin{array}{l}\mathrm{c} \\
\mathrm{d} \\
\mathrm{e} \\
\mathrm{f} \\
\mathrm{g} \\
\mathrm{h}\end{array}$ & $\begin{array}{l}0.1139 \\
0.0595 \\
0.0633 \\
0.1216 \\
0.5785 \\
0.0727\end{array}$ & $\begin{array}{l}0.0018 \\
0.0005 \\
0.0005 \\
0.009 \\
0.2767 \\
0.7197\end{array}$ & $\begin{array}{c}80 \mathrm{C}=353 \mathrm{~K} \\
1.00 \mathrm{bar}\end{array}$ & $\mathrm{H}_{6}=-128,086$ & $m_{6}{ }_{6}=-12: 14 \mathrm{E} 6$ \\
\hline
\end{tabular}

$\begin{array}{lllcl}7(G) & 71.60 & \mathrm{~g} & 0.0909 & 44 \mathrm{C}=317 \mathrm{~K} \\ 35.532 & \mathrm{~h} & 0.9091 & 1.00 \text { bar }\end{array} \quad \mathrm{H}_{8}=-21,399 \quad \mathrm{~m}_{8} \mathrm{H}_{8}=-0.7604 \mathrm{E} 6$

$\begin{array}{llll}11 & (\mathrm{~L}) \\ 41.455 & \mathrm{~g} & 1.0000 & \begin{array}{c}44 \mathrm{C}=317 \mathrm{~K} \\ 1.00 \text { bar }\end{array}\end{array} \quad \mathrm{H}_{11}=-281,281 \quad \mathrm{~m}_{11} \mathrm{H}_{11}=-11.66 \mathrm{E} 6$

\begin{tabular}{|c|c|c|c|c|c|c|}
\hline $14(\mathrm{~L})$ & $\begin{array}{l}2004.9 \\
17.772\end{array}$ & $\begin{array}{l}c \\
d \\
e \\
f\end{array}$ & $\begin{array}{l}0.3333 \\
0.1667 \\
0.1667 \\
0.3333\end{array}$ & $\begin{array}{c}470 \mathrm{C}=743 \mathrm{~K} \\
1.00 \mathrm{bar}\end{array}$ & $\mathrm{H}_{14}=-146,411$ & $\mathrm{~m}_{14}{ }^{\mathrm{H}}{ }_{14}=-2.6002 \mathrm{E} 6$ \\
\hline
\end{tabular}

\begin{tabular}{|c|c|c|c|c|c|}
\hline$\underline{15}(\mathrm{~L})$ & $\begin{array}{l}596.69 \\
5.289\end{array}$ & ( " ) & $\begin{array}{c}35 \mathrm{C}=308 \mathrm{~K} \\
1.00 \mathrm{bar}\end{array}$ & $\mathrm{H}_{15}=-182,691$ & $\mathrm{~m}_{15}{ }^{\mathrm{H}} 15=-3.247 \mathrm{E} 6$ \\
\hline
\end{tabular}


Table 2. REACTOR SPECIFICATIONS

Item Description

1) Type Fliuidized Bed w/top entry solar energy;

w/cyclone separator on discharge

2) Catalyst

$124 \mathrm{~kg} ; 3$ w\% $\mathrm{V}_{2} \mathrm{O}_{5}$ on $\mathrm{SiO}_{2}$ support

60-80 micron particle size; $300 \mathrm{~m}^{2}$ surf. area

catalyst bed density $=0.431 \mathrm{~g} / \mathrm{cm}^{3}=26.91 \mathrm{bm} / \mathrm{ft}^{3}$

3) Bed dimensions $1.134 \mathrm{~m}$ dia $\times 0.284 \mathrm{~m} \mathrm{ht}$.

volume $=0.2863 \mathrm{~m}^{3}=10.11 \mathrm{ft}^{3}$

c.s. area $=1.010 \mathrm{~m}^{2}=10.87 \mathrm{ft}^{2}$

4) Vessel dimens. 45" i.d. $x 1^{\prime}$ ' $s$ to $s$; $2^{\prime}$ dia top quartz window, catalyst bed support grid; conical bottom;

two-stage cyclone separator

- Operating Conditions.

entrance

exit

5) Flow rate $\quad 71.063 \mathrm{kgmols} / \mathrm{h} \quad 94.763$

$2822.7 \mathrm{~kg} / \mathrm{h}$

$=$

6) $\mathrm{T}, \mathrm{P} \quad 185 \mathrm{C}, 1.70$ bar $400 \mathrm{C}, 1.50$ bar

7) Gas density

$1.774 \mathrm{~kg} / \mathrm{m}^{3}$

$0.7989 \mathrm{~kg} / \mathrm{m}^{3}$

8) Space velocity

$22.76 \mathrm{~kg} / \mathrm{h} \mathrm{kg}$ cat.

9) Mass velocity $2795 \mathrm{~kg} / \mathrm{h} \mathrm{m}^{2}$

10) Min. fluidization $1982 \mathrm{~kg} / \mathrm{h} \mathrm{m}^{2}$ mass velocity

11) Q solar* $0.604 \mathrm{~kJ} / \mathrm{h}=168 \mathrm{~kW}$

12) Material of construction: carbon steel shell, catalyst grid support, hi-temp refractory cement lining, top quartz window; carbon steel (w/liner) cyclone

13) Estimated Cost:

$\$ 31,210$

* Based on thermodynamic requiremeni 


\subsection{EQUIPMENT SIZING AND COST}

Specifications and costs for the individual pieces of equipment are presented in Tables 4-7 inc1. Costs rere estimated using the functions and charts given by Guthrie [5] based on 1974 data. Fhich vere escalated to 1989. The cost escalation index (see Appendix C) ras calculated by. $I(1974)=202.5$. $I(1989)=391.0$. giving $I c=391.0 / 202.5=$ 1.931.

The costs by equipment catagory are summarized in Table 3, as follors.

Table 3. SUMMARI OF EQUIPMENT COSTS

(fresh feed $=7.056$ million $\mathrm{kg} / \mathrm{y}=15.56 \mathrm{million} 1 \mathrm{bm} / \mathrm{y}$ )

catagor

1) Reactor v/2-stage Cyclone

2) Heat exchangers

3) Distillation columns

4) Accumulators (A1.5d storage)

5) Pumps vith spares

6) Storage tanks

7) Process Instruments \& Controls (15\%)

8) Computer (data logging contro1)

9) Catalyst, initial charge number Cost (\$)

(1)

31,210

(11)

126,435

(3)

118,230

(4)

61,170

(7)

18,100

(4)

191.100

$(546.245)$

81,937

(1)

110,000

(1)

1.000 
Table 4 - HEAT EXCHANGER SUMMARY

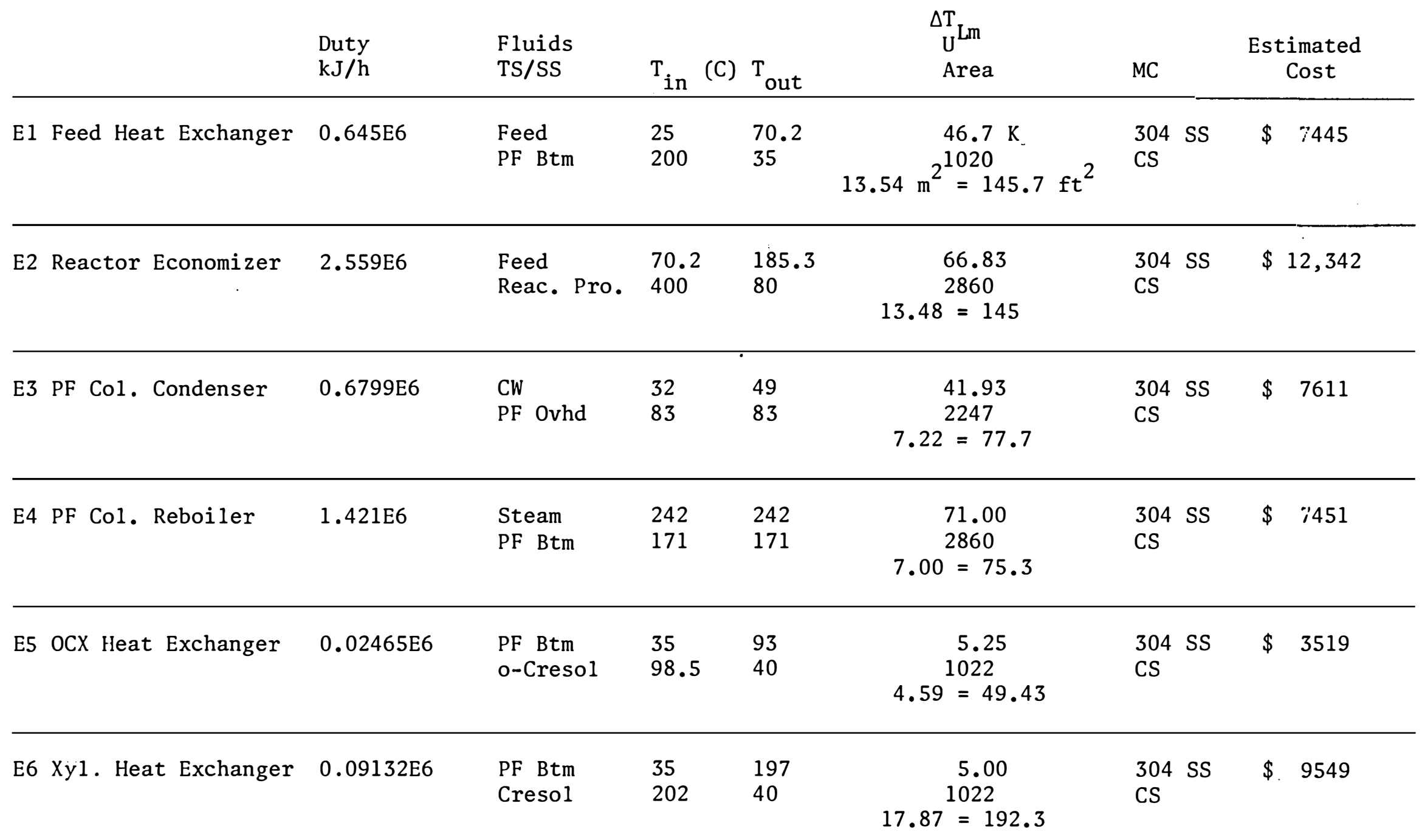


Table 4 - HEAT EXCHANGER SUMMARY (continued)

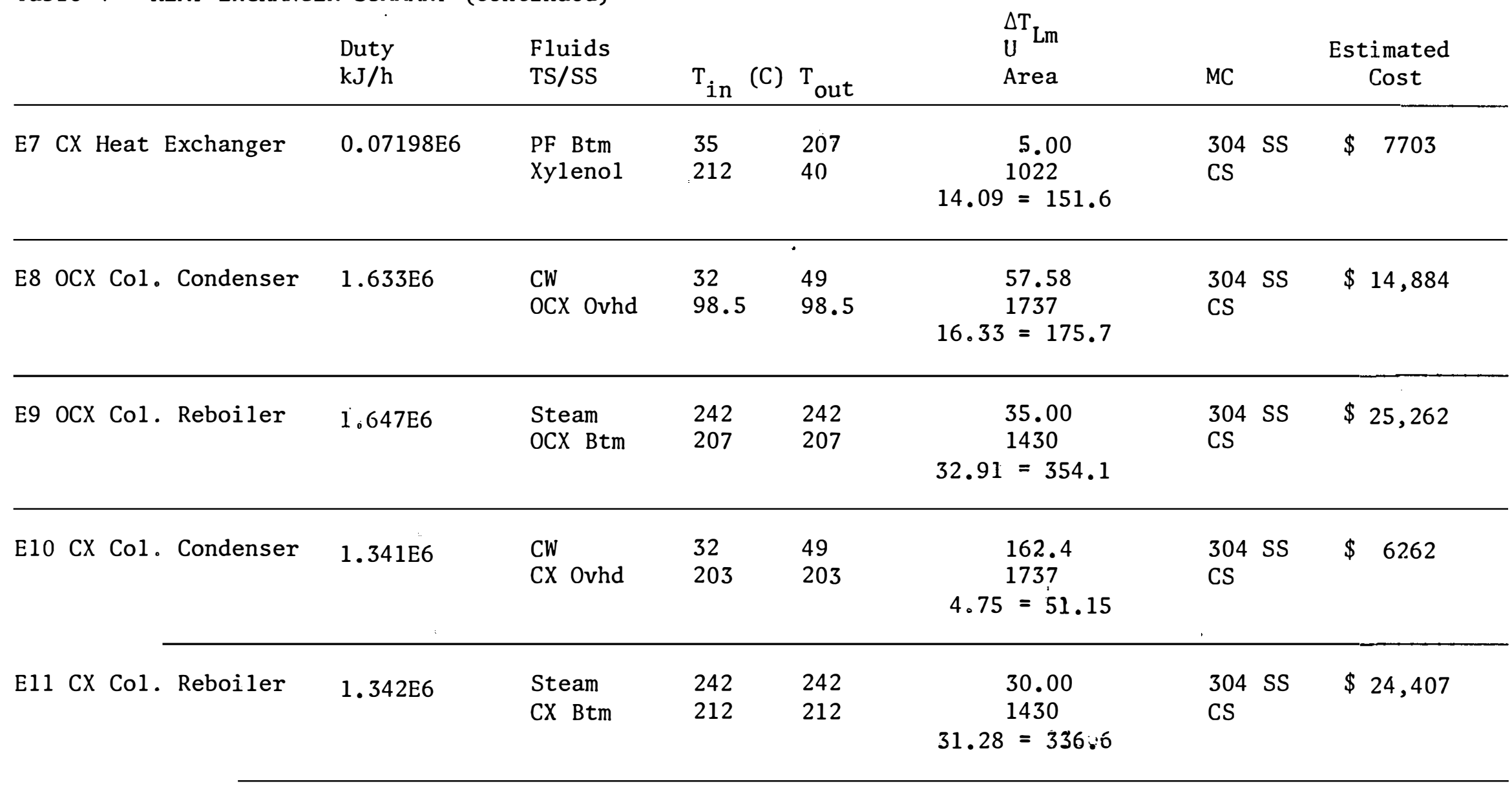


Table 5a. PF Distillation Column

Feed stream 6; overhead stream $\underline{7}$; bottoms stream $\underline{12}$

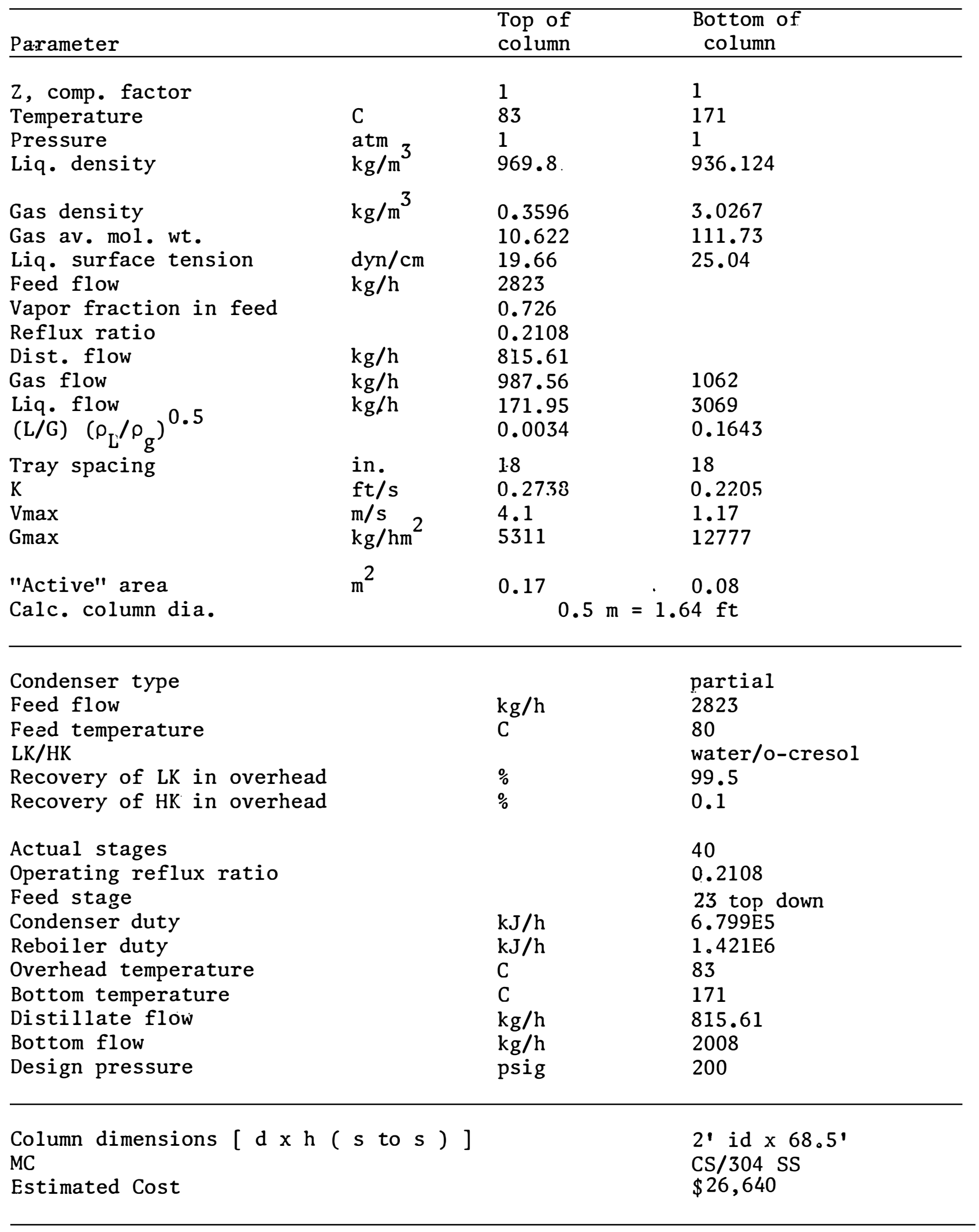


Table 5b. OCX Distillation Column

Feed stream 26; overhead stream 27; bottoms stream 29

\begin{tabular}{|c|c|c|c|}
\hline Parameter & & $\begin{array}{l}\text { Top of } \\
\text { column }\end{array}$ & $\begin{array}{l}\text { Bottom of } \\
\text { column }\end{array}$ \\
\hline $\begin{array}{l}\text { Z, comp. factor } \\
\text { Temperature } \\
\text { Pressure } \\
\text { Liq. density }\end{array}$ & $\begin{array}{l}\mathrm{C} \\
\mathrm{atm} \\
\mathrm{kg} / \mathrm{m}^{3}\end{array}$ & $\begin{array}{l}0.9747 \\
98.5 \\
1 \\
965.488\end{array}$ & $\begin{array}{l}1 \\
207 \\
1 \\
917.56\end{array}$ \\
\hline $\begin{array}{l}\text { Gas density } \\
\text { Gas av. mol. wt. } \\
\text { Liq. surface tension } \\
\text { Feed flow } \\
\text { Vapor fraction in feed } \\
\text { Reflux ratio } \\
\text { Dist. flow } \\
\text { Gas flow } \\
\text { Liq. flow } \\
(\mathrm{L} / \mathrm{G})\left(\rho_{\mathrm{L}} / \rho_{\mathrm{g}}\right)\end{array}$ & $\begin{array}{l}\mathrm{kg} / \mathrm{m}^{3} \\
\mathrm{dyn} / \mathrm{cm} \\
\mathrm{kg} / \mathrm{h}\end{array}$ & $\begin{array}{l}2.802 \\
105.061 \\
19.66 \\
597.367 \\
0.00 \\
12.68 \\
189.735 \\
2073.2 \\
1883.4 \\
0.0489\end{array}$ & $\begin{array}{l}2.8848 \\
115.124 \\
25.04\end{array}$ \\
\hline $\begin{array}{l}\text { Tray spacing } \\
\text { K } \\
\text { Vmax } \\
\text { Gmax }\end{array}$ & $\begin{array}{l}\text { in } \\
\mathrm{ft} / \mathrm{s} \\
\mathrm{m} / \mathrm{s} \\
\mathrm{kg} / \mathrm{hm}^{2}\end{array}$ & $\begin{array}{l}18 \\
0.2573 \\
1.38 \\
13883\end{array}$ & $\begin{array}{l}18 \\
0.2510 \\
1.35 \\
14058\end{array}$ \\
\hline $\begin{array}{l}\text { "Active" area } \\
\text { Calc. column dia. }\end{array}$ & $m^{2}$ & $\begin{array}{ll}0.15 & \\
& 0.4\end{array}$ & $\begin{array}{l}0.15 \\
.44 \mathrm{ft}\end{array}$ \\
\hline $\begin{array}{l}\text { Condenser type } \\
\text { Feed flow } \\
\text { Feed temperature } \\
\text { LK/HK } \\
\text { Recovery of LK in overhead } \\
\text { Recovery of HK in overhead } \\
\text { Actual stages } \\
\text { Operating reflux ratio } \\
\text { Feed stage } \\
\text { Condenser duty } \\
\text { Reboiler duty } \\
\text { Overhead temperature } \\
\text { Bottom temperature } \\
\text { Distillate flow } \\
\text { Bottom flow } \\
\text { Design pressure }\end{array}$ & & $\begin{array}{l}\mathrm{kJ} / \mathrm{h} \\
\mathrm{kJ} / \mathrm{h} \\
\mathrm{C} \\
\mathrm{C} \\
\mathrm{kg} / \mathrm{h} \\
\mathrm{kg} / \mathrm{h} \\
\mathrm{psig}\end{array}$ & $\begin{array}{l}\text { total } \\
597.4 \\
168 \\
\text { o-cresol/m-cresol } \\
99 \\
0.1 \\
82 \\
12.684 \\
50 \text { top down } \\
1.633 \mathrm{E} 6 \\
1.647 \mathrm{E} 6 \\
99 \\
207 \\
189.7 \\
407.6 \\
200\end{array}$ \\
\hline $\begin{array}{l}\text { Column dimensions }[\mathrm{d} \times \mathrm{h} \\
\text { MC } \\
\text { Estimated Cost }\end{array}$ & s to s ) ] & & $\begin{array}{l}2^{\prime} \text { id } \times 131.5^{\prime} \\
\mathrm{CS} / 304 \mathrm{SS} \\
\$ 47,890\end{array}$ \\
\hline
\end{tabular}


Table 5c. CX Distillation Column

Feed stream 30; overhead stream 31; bottoms stream $\underline{33}$

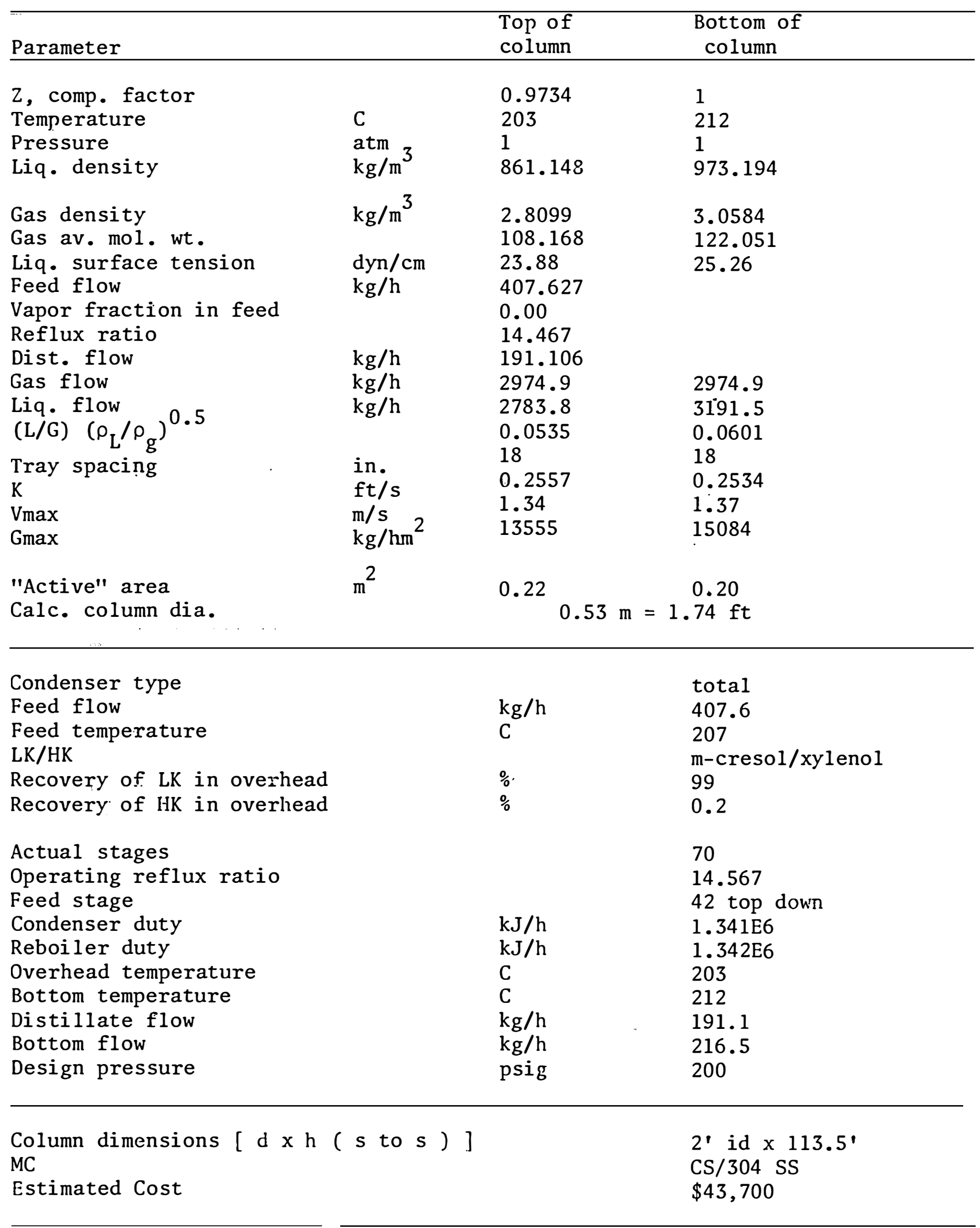


Table 6. PUMP SUMMARY

(Centrifugal, stainless; costs for pump + spare + motor)

\begin{tabular}{lccccc}
\hline Pump & $\begin{array}{c}\text { Inlet } \\
\text { stream }\end{array}$ & $\begin{array}{c}\text { Capacity } \\
(\mathrm{gpm})\end{array}$ & $\Delta \mathrm{P}(\mathrm{psi})$ & BHP & Cost $(\$)$ \\
\hline P1 & 1 & 16 & 500 & 5.62 & 5973 \\
P2 & $\underline{9}$ & 3 & 135 & 0.29 & 1928 \\
P3 & $\underline{16}$ & 3 & 135 & 0.24 & 1892 \\
P4 & from E8 & 2 & 135 & 0.86 & 2109 \\
P5 & $\underline{29}$ & 9 & 135 & 0.16 & 1904 \\
P6 & from E10 & 13 & 135 & 1.25 & 2402 \\
P7 & $\underline{33}$ & 1 & 135 & 0.08 & 1908 \\
\hline
\end{tabular}

Total $\$ 18,100$

Table 7. STOARAGE TANK SUMIARY

(Based on approximate 10-day storage time, except 5 days for methanol)

\begin{tabular}{llcccc}
\hline Tank & Substance & $\begin{array}{c}\text { Density } \\
(\mathrm{kg} / \text { liter) }\end{array}$ & $\begin{array}{c}\text { Capacity } \\
(\mathrm{U} . \mathrm{S} \text {.Gallons) }\end{array}$ & MC & $\begin{array}{c}\text { Estimated } \\
\text { Cost(1988) }\end{array}$ \\
\hline T1 & Benzene & 0.879 & 67,000 & CS & $\$ 71,900$ \\
T2 & Methanol & 0.792 & 75,000 & CS & 80,500 \\
T3 & o-Cresol & 1.048 & 12,000 & CS & 12,900 \\
T4 & m,p-Cresol & 1.035 & 12,000 & CS & 12,900 \\
T5 & Xylenol & 1.1695 & 12,000 & CS & 12,900 \\
\hline
\end{tabular}

Total $\$ 191,100$ 


\subsection{ESTIMATED CAPITAL AND OPERATING COSTS}

The methods used to calculate the process economics follov standard chemical engineering procedures as presented by Guthrie I5L Peters and Timmerhaus [6 $\mathrm{L}$ and Perry's Handbook [7].

Table 8. Estimated Capital Cost

(fresh feed $=7.056$ million $\mathrm{kg} / \mathrm{g}=15.56$ million $1 \mathrm{bm} / \mathrm{g}$ ) Item

factor

$\$ 1000$

A. Equipment (Table 3)

739.2

B. Interconnecting piping, flanges, fittings.

110.9 valves

(15\% A)

C. Assemb1y and Installation of Equipment purchasing, materials, labor and supervision

foundations and structures running pipe, steam tracing, insulation. painting electrical

$(40 \%$ i)

295.7

221.8

$(30 \times \Delta)$

(20\% $\Delta) \quad 147.8$

(10₹ $\Delta) \quad 73.9$

(1589.3)

D. Design and Engineering

E. BASE STSTEN COST (BSC)

$(8 \times A+B+C)$

127.1

1716.4

F. Contractors fee

$(8 \times \mathrm{BSC}) \quad 137.3$

G. Contingency

(15\% BSC) 257.5

H. TOTAL IHSTALLED PLAHT COST

$\$ 2.111 \mathrm{E6}$

It has been shovn by a number of authors [5,6.7] that plant capital cost can be correlated by a 6-tenths-pover relationship. For this plant $\left[F=7.056 \times 10^{6} \mathrm{~kg} / 7\right.$. Ic $\left.(1989)=1.931\right]$ the equation becomes,

$C(\$)=$ Co IC F $F^{0.60}=(85.029)$ IC $F^{0.60}$

Thich can be used as the capital cost scaling-equation for different size plants. For example, for a plant of $\mathrm{F}=14 \mathrm{E} 6 \mathrm{~kg} / \mathrm{F}$ the estimated cost is \$ $3.184 \mathrm{E} 6$.

The estimated plant operating cost is presented in Table 9 . It is assumed that the plant is a process unit rithin an existing chemical plant vhere necessary utilities and other services are available. 
Table 9. Plant Operating Cost

( 2500 sun hours; 8400 operating $h / g$ )

Item $\$ / Y$

1) Ray Materials (benzene, methanol, supplies. $1.648 \mathrm{E6}$ make-up catal pst)

2) Operating labor supervision (3-operators, 0.5292 1-technicial, 1/2 foreman. per shift)

3) Utilities (nat'1 gas, steam, cooling vater, elec- 0.09125 (ric pover)

4) Maintenance \& Repair (7\% of capital $\operatorname{cost} / \bar{\gamma}$ ) 0.1478

5) Plant overhead (75x operating labor) 0.3969

6) Plant G/A (25\% operating labor) 0.1323

7) Depreciation (15 y life, loan payment) 0.1407

8) Interest on borro $\mathrm{y}$ capital $(10 \% / 7,15 \%$, on 0.2111 declining balance)

9) Iaxes and Insurance ( $3 \%$ of capital cost/7) 0.0633

10) TOTAL OPERATIHG COST \$ 3.361 E6

11) Unit COST of PRODUCTS SOLD (3.361/11.443) $\begin{gathered}\$ 0.2937 / 1 \mathrm{bm} \\ \$ 0.6475 / \mathrm{kg}\end{gathered}$

The profitability analysis and break-eren capacity nov can be calculated! 


\subsection{PROFITABILITY ANALYSIS}

Hov it is necessary to determine Fhether or not the base case plant size rould be a profitable renture. First, the plant income from sale of products can be calculated, as shorn in Table 10.

Table 10. Plant Income from Products

\begin{tabular}{|c|c|c|c|}
\hline product & $1 \mathrm{bm} / \mathrm{Y}$ & $\$ / 1 \mathrm{bm}$ & $\$ / Y$ \\
\hline 1) hydrogen & 0.3948 E6 & 0.0307 & 12120 \\
\hline 2) o-Cresol & $3.530 \mathrm{E} 6$ & 0.580 & 2.047 E6 \\
\hline 3) m,p-cresols & $3.350 \mathrm{E} 6$ & 0.820 & 2.895 E6 \\
\hline 4) mixed IYlenols & $3.988 \mathrm{E} 6$ & 0.580 & $2.313 \mathrm{E} 6$ \\
\hline Totals & $11.443 \mathrm{E} 6$ & & $7.267 \mathrm{E} 6$ \\
\hline \multicolumn{3}{|c|}{ 6) Average unit income $(7.267 / 11.443)$} & $\begin{array}{l}\$ 0.6351 / 16 \\
\$ 1.400 / \mathrm{kg}\end{array}$ \\
\hline
\end{tabular}

Comparing the average $\$ / 1 \mathrm{bm}$ income vith the cost/1bm (Iable 9 ) it is obvious that the base case is beyond break-eren, and the capacity vill have to be reduced to find the break-even point. The operating cost items in Iable 9 can be vritten in equation form and programed as a runction of plant capacity: some items are directly proportional and some less than proportional to size (e.g. capital cost). vhile others are constant. Table 11 presents a print-out for determination of the first-fear break- even point, giving the folloving values:
1) Fresh Feed
$(7.056 \mathrm{E6})(0.2341)=$
$1.652 \mathrm{E} 6 \mathrm{~kg} / \mathrm{7}$
2) Products
$(5.192 \mathrm{E6})(0.2341)=$
$1.215 \mathrm{E} 6 \mathrm{~kg} / \mathrm{\gamma}$
3) Capital Cost
$\$ 0.8833$ E6
4) Op-Cost \& Income
$\$ 1.701 \mathrm{E} 6 / \mathrm{Y}$

The qualifier "first-year" is used because the interest payment declines each year as the principal is reduced.

Finally. the vork described herein permits the folloving evaluative conclusions to be dravn for the process:

1. -a plant producing greater than $2.679 \mathrm{E} 61 \mathrm{bm} / \mathrm{7}=1.215 \mathrm{E} 6 \mathrm{~kg} / \mathrm{7}$ of products rould be a profitable venture.

2. -the configuration of the process indicates the equipment complezity, vhich directiy arfects the capital in vestment required.

3. -the major item of operating cost is rav materials - 49\% of total.

4. - the report provides a basis for comparison vith the Conventional Catalytic Process described in the first report. Because of 10 ver rav 
Table 11. Calculation of First-Year Break-Even Capacity

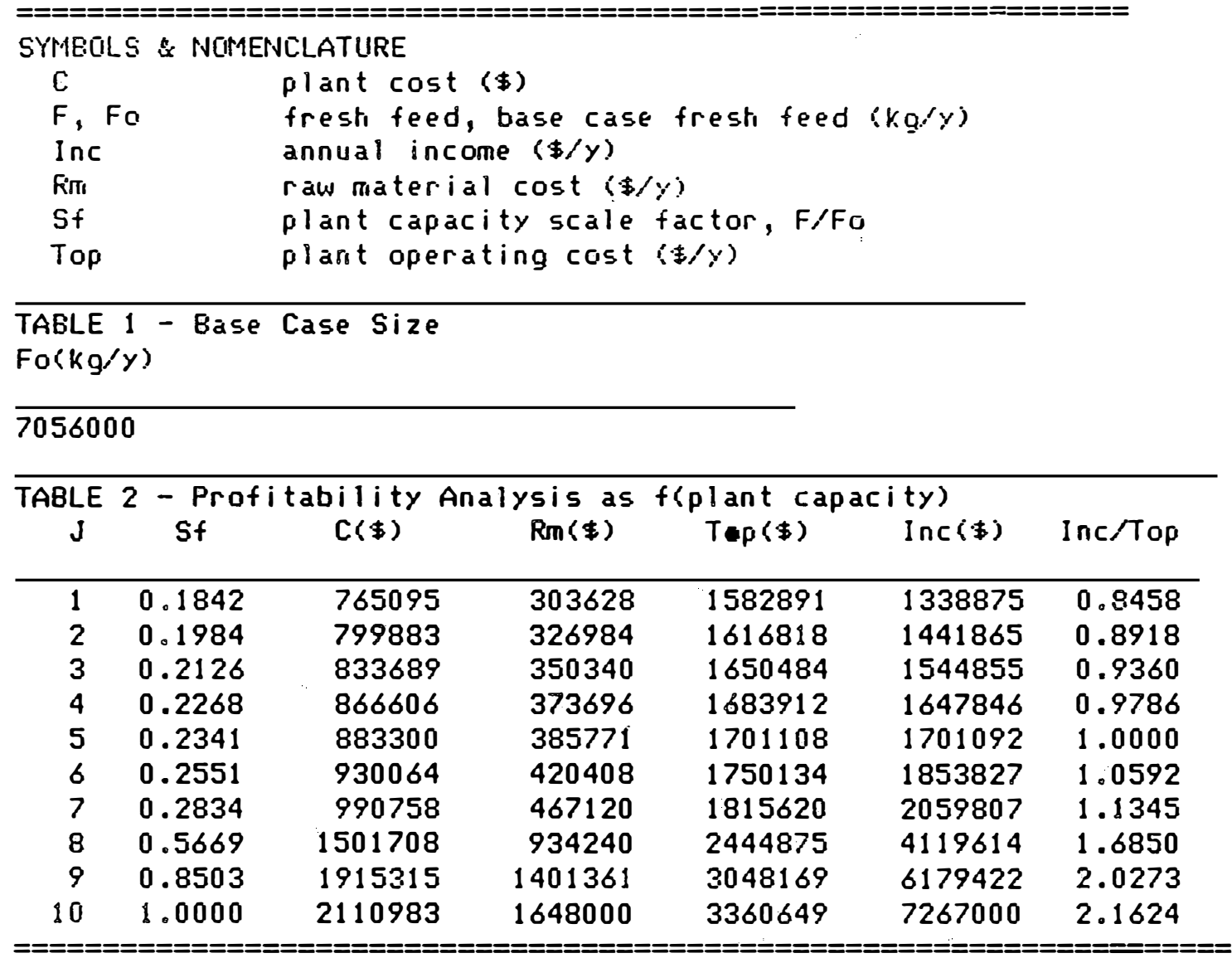

Dut of Data

ENO OF CALCLLATION (9-26-89)

material cost and higher income, the break-eren size vas significantly lover for the Solar-Catalytic Process.

5. - the capital cost estimate is for the chemical process equipment. and does not include the cost of equipment to deliver the solar energy to the top of the reactor. Assuming the total capital cost rould double. vhich is unlikely. vhen the solar energy equipment is included, the conclusion in favor of the photo-catalptic process vould not change. 


\section{REFERENCES CITED}

[1] COADE-CHEMSTATIONS Inc., Engineering Software; 10375 Richmond Ave, Suite 1225; Houston, Tezas 77042: CHEMCAD II - Process Flowsheet Simulator May 1989.

[2] R.M.Guthrie, Process Plant Estimating Evaluation and Control. Craftsman Book Company of America, Solana Beach, CA 92075, (1974).

[3] M.S. Peters and R.D.Timmerhaus, plant Design and Economics for Chemical Engineers, third edition, McGraw-Hill Book Company, NY (1980).

[4] F.A. Holland, F.A. Matson, and J.R. Milkinson, Section 25: Process Economics - Perry's Chemical Engineers Handbook. sixth edition; McGraw-Hill Book Company, NY (1984).

[5] Schnell Publishing Co., Inc., Chemical Marketing Reporter, 19 June 1989 issue. 
A1 - Benzene, $\mathrm{C}_{6} \mathrm{H}_{6}$

1) Molecular Weight, $M$

78.113

2) $\mathrm{T}_{\mathrm{c}}(\mathrm{K}), \mathrm{P}_{\mathrm{c}}(\mathrm{atm}), \mathrm{V}_{\mathrm{c}}\left(\mathrm{cm}^{3} / \mathrm{gmol}\right), \mathrm{Z}_{\mathrm{c}}$

$562.16,48.34,258.94,0.274$

3) Acentric factor; dipole moment

$\omega=0.209, \mu=0.0 \mathrm{D}$

4) $T_{t p}(K), T_{b}(K)$

$278.66,353.3$

5) At $293.16 \mathrm{~K}, \rho_{1}\left(\mathrm{~g} / \mathrm{cm}^{3}\right)$

0.879

6) At $298.16 \mathrm{~K}$

$\mathrm{Cp}(1)$

$\mathrm{Cp}^{0}$

$\mathrm{S}^{0} \quad \Delta \mathrm{H}^{\mathrm{V}}$

$\Delta \mathrm{H}_{\mathrm{f}}{ }^{0} \quad \Delta \mathrm{G}_{\mathrm{f}}{ }^{0}$

a)

32.11

$19.52 \quad 64.34$

7.352

19.82

30.99 (cals/gmol, K) (kcals/gmol)

t) 134.35

81.71

269.33 (kJ/kgmol, K)

30,761

(kJ/kgmol)

\section{Equations}

1) Liquid volume, $\mathrm{v}_{1}=12.26\left(5.7+3 \mathrm{~T}_{\mathrm{r}}\right),\left(\mathrm{cm}^{3} / \mathrm{gmol}\right)$

2) Liquid density, $\ln \rho_{1}=-0.024098+1.344347\left[1+(1-\mathrm{T} / 0.56216 \mathrm{E} 3)^{0.27357 \text {. }}\right.$ $\left(\mathrm{kgmol} / \mathrm{m}^{3}\right)$

3) Vapor pressure: In $\mathrm{P}($ torr $)=16.1753-2948.78 /(\mathrm{T}-44.563)$

$$
\ln \mathrm{P}(\mathrm{atm})=10.0114-3291.87 / \mathrm{T}-84994.9 / \mathrm{T}^{2}
$$

4) Mole $\mathrm{Cp}^{0} / \mathrm{R}=-2.69988+4.83994 \mathrm{E}-2 \mathrm{~T}-2.06081 \mathrm{E}-5 \mathrm{~T}^{2}$

5) Mole $C_{p}(1)=32.3793+0.342 \mathrm{~T},(\mathrm{~kJ} / \mathrm{kgmol}, \mathrm{K})$

6) $\Delta \mathrm{H}^{\mathrm{V}}=\mathrm{RT}^{2} \Delta \mathrm{Z}(\mathrm{d} \ln \mathrm{P} / \mathrm{dT})$

7) Surface tension, $\sigma=0.07195\left(1-\mathrm{T}_{\mathrm{r}}\right)^{1.2389},(\mathrm{~N} / \mathrm{m})$

8) Viscosity, vs: $\log$ vs $(c p)=545.64(1 / T-1 / 265.34)$ 
APPENDIX A (çontinued)

A2 - Methanol, $\mathrm{CH}_{3} \mathrm{OH}$

1) Molecular Weight, M $\quad 32.042$

2) $\mathrm{T}_{\mathrm{c}}(\mathrm{K}), \mathrm{P}_{\mathrm{c}}(\mathrm{atm}), \mathrm{V}_{\mathrm{c}}\left(\mathrm{cm}^{3} / \mathrm{gmol}\right), \mathrm{Z}_{\mathrm{c}} \quad 512.58,79.9,117.8,0.222$

3) Acentric factor; dipole moment $\omega=0.5656, \mu=1.70 \mathrm{D}$

4) $\mathrm{T}_{\mathrm{tp}}(\mathrm{K}), \mathrm{T}_{\mathrm{b}}(\mathrm{K}) \quad 175.7,337.8$

5) At $293.16 \mathrm{~K}, \rho_{1}\left(\mathrm{~g} / \mathrm{cm}^{3}\right) \quad 0.792$

6) At $298.16 \mathrm{~K} \quad \mathrm{Cp}(1) \cdot \mathrm{Cp}^{0} \quad \mathrm{~S}^{0} \quad \Delta \mathrm{H}^{\mathrm{V}} \quad \Delta \mathrm{H}_{\mathrm{f}}^{0} \quad \Delta \mathrm{G}_{\mathrm{f}}^{0}$
a)
$46.52 \underset{(c a l s / g m o l, K)}{10.49} 57.29$
$\begin{array}{lll}8.426 & -48.08 & -38.84 \\ (\mathrm{kcal} / \mathrm{gmol}) & -30\end{array}$
b)

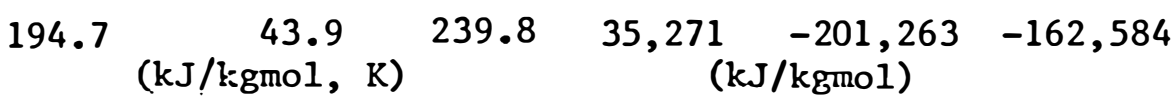

\section{Equations}

1) Liquid volume, $\mathrm{v}_{1}=5.4628\left(5.7+3 \mathrm{~T}_{\mathrm{r}}\right),\left(\mathrm{cm}^{3} / \mathrm{gmol}\right)$

2) Liquid density, $\ln \rho_{1}=0.18706+1.62055\left[1+(1-\mathrm{T} / 0.51263 E 3)^{0.17272 j}\right.$,

$\left(\mathrm{kgmol} / \mathrm{m}^{3}\right)$

3) Vapor pressure: In $\mathrm{P}($ torr $)=18.5097-3593.39 /(\mathrm{T}-35.225)$

$\ln \mathrm{P}(\mathrm{atm})=11.9921-3679.33 / \mathrm{T}-126059 / \mathrm{T}^{2}$

4) Mole $\mathrm{Cp}^{0} / \mathrm{R}=1.85019+0.0124255 \mathrm{~T}-3.49129 \mathrm{E}-6 \mathrm{~T}^{2}$

5) Mole $\mathrm{Cp}_{\mathrm{p}}(1)=-39.9665+0.787208 \mathrm{~T},(\mathrm{~kJ} / \mathrm{kgmol}, \mathrm{K})$

6) $\Delta \mathrm{H}^{\mathrm{V}}=\mathrm{RT}^{2} \Delta \mathrm{Z}(\mathrm{d} \ln \mathrm{P} / \mathrm{dT})$

7) Surface tension, $\sigma=0.04327\left(1-\mathrm{T}_{\mathrm{r}}\right)^{0.7676},(\mathrm{~N} / \mathrm{m})$

8) Viscosity, vs: $\log$ vs $(c p)=555.3(1 / T-1 / 263.64)$ 
APPENDIX A (continued)

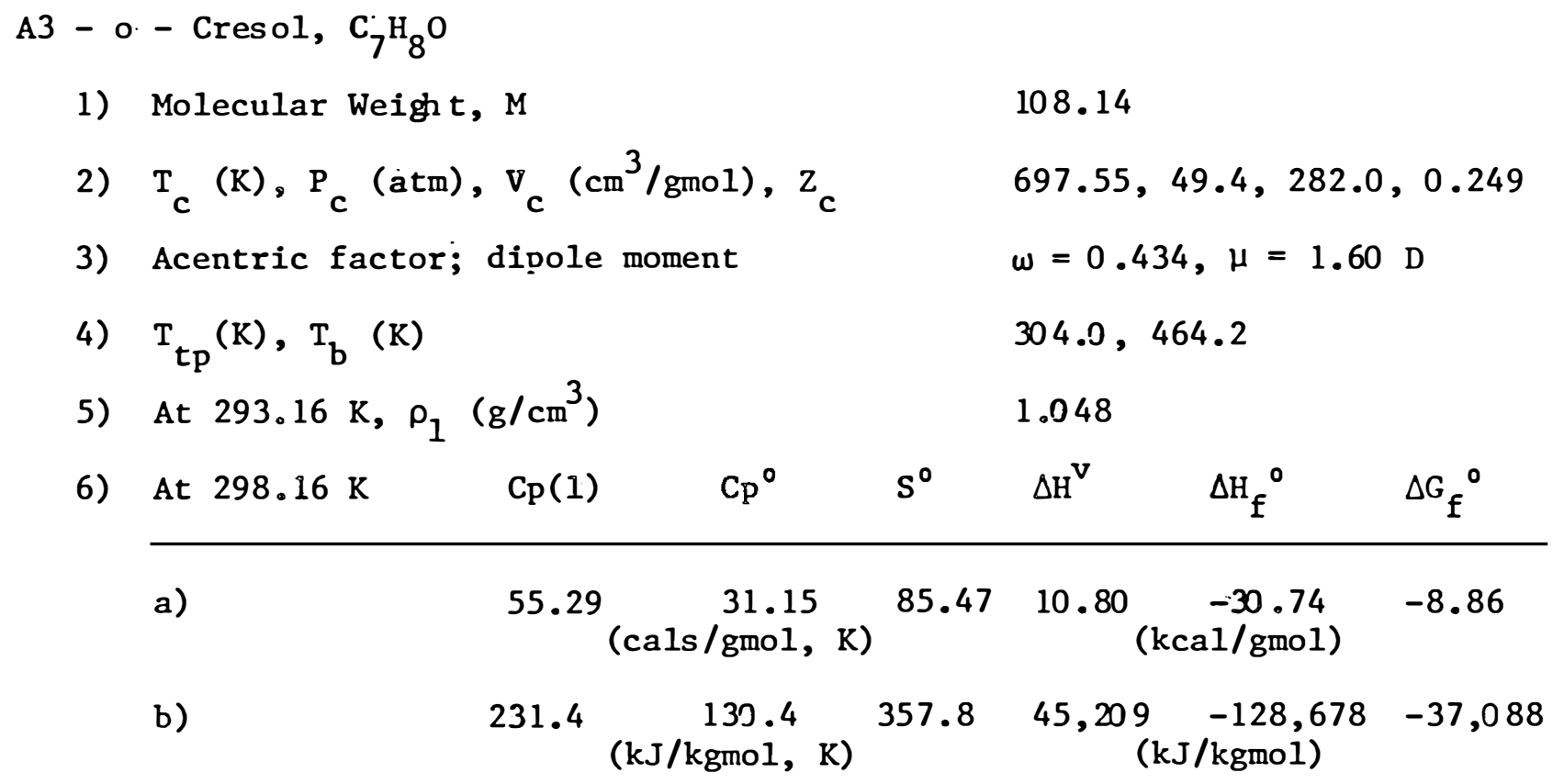

Equations

1) Liquid volume; $v_{1}=14.9288\left(5.7+3 \mathrm{~T}_{\mathrm{r}}\right),\left(\mathrm{cm}^{3} / \mathrm{gmol}\right)$

2) Liquid density, $\ln \rho_{1}=0.58912+1.18685\left[1+(1-\mathrm{T} / 0.69755 \mathrm{E} 3)^{0.3099}\right]$, $\left(\mathrm{kgmol} / \mathrm{m}^{3}\right)$

3) Vapor pressure: In $\mathrm{P}($ torr $)=16.2829-3552.74 /(\mathrm{T}-95.975)$

$$
\ln \mathrm{P}(\mathrm{atm})=11.1411-4406.52 / \mathrm{T}-351528 / \mathrm{T}^{2}
$$

4) Mole $\mathrm{C}_{\mathrm{P}} \% / \mathrm{R}=1.40132+0.0552621 \mathrm{~T}-2.28792 \mathrm{E}-5 \mathrm{~T}^{2}$

5) Mole $\left.\mathrm{C}_{\mathrm{p}}(1)=559.336-1.86259 \mathrm{~T}+1\right) .2258292 \mathrm{E}-2 \mathrm{~T}^{2},(\mathrm{~kJ} / \mathrm{kgmol}, \mathrm{K})$

6) $\Delta \mathrm{H}^{\mathrm{V}}=\mathrm{RT}^{2} \Delta \mathrm{Z}(\mathrm{d} \ln \mathrm{P} / \mathrm{dT})$

7) Vis cosity, vs: $\log$ vs $(c p)=1785.6(1 / T-1 / 370.75)$ 
APPENDIX A (continued)

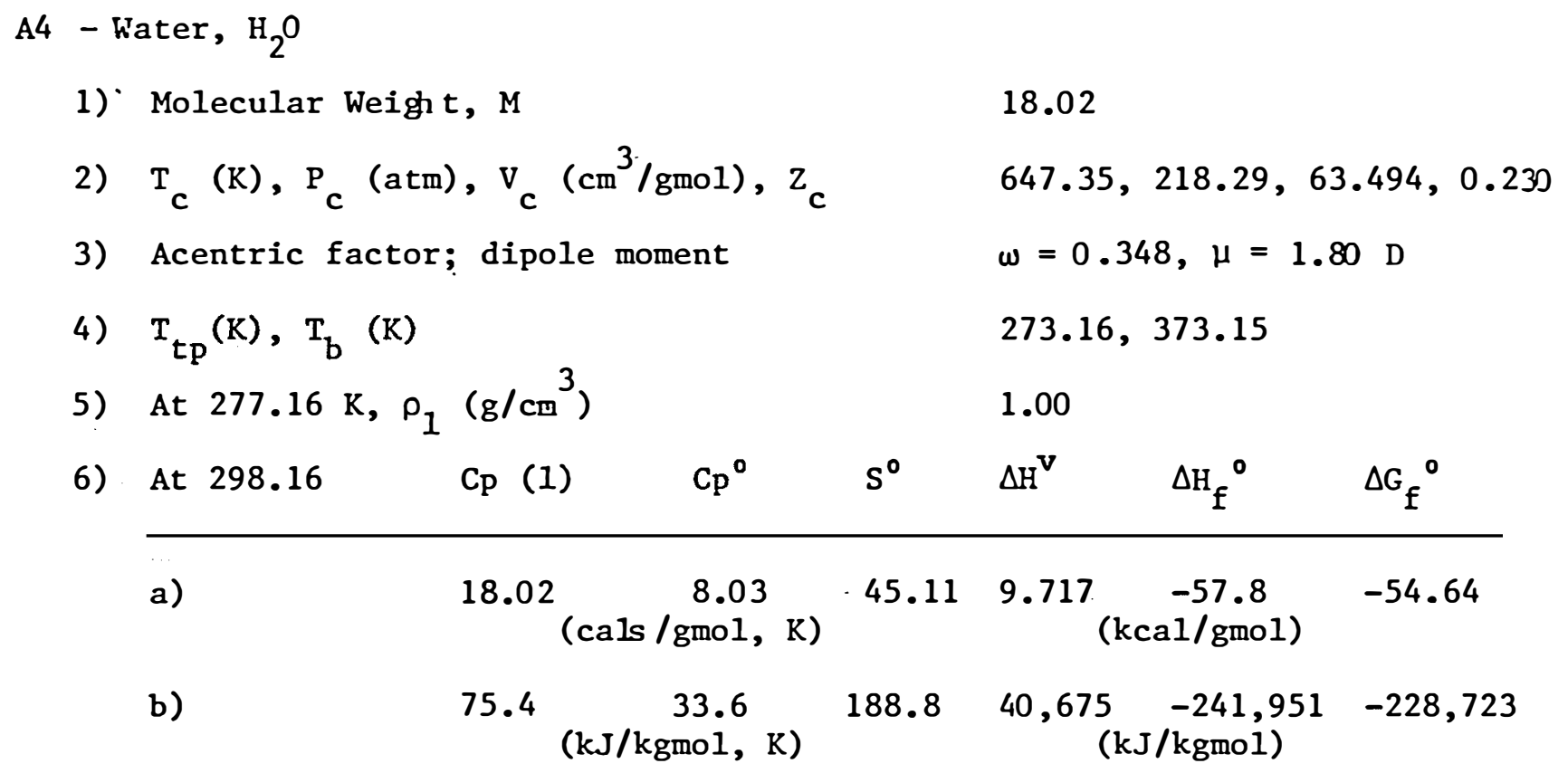

\section{Equations}

1) Liquid volume, $\mathrm{v}_{1}=2.552\left(5.7+3 \mathrm{~T}_{\mathrm{r}}\right),\left(\mathrm{cm}^{3} / \mathrm{gmol}\right)$

2) Liquid density, $\ln \rho_{1}=1.52903+1.33888\left[1+(1-\mathrm{T} / 0.64729 \mathrm{E} 3)^{0.23072}\right]$, $\left(\mathrm{kgmol} / \mathrm{m}^{3}\right)$

3) Vapor pressure: In $P($ torr $)=18.3036-3816.44 /(t-46.13)$

$$
\ln \mathrm{P}(\mathrm{atm})=11.6572-3761.58 / \mathrm{T}-218339 / \mathrm{T}^{2}
$$

4) Mole $\mathrm{CP}_{\mathrm{P}} \% \mathrm{R}=2.37293+0.0160161 \mathrm{~T}-7.40155 \mathrm{E}-6 \mathrm{~T}^{2}$

5) Mole $\mathrm{C}_{\mathrm{p}}(1)=32.4953+0.124601 \mathrm{~T},(\mathrm{~kJ} / \mathrm{kgmol}, \mathrm{K})$

6) $\Delta \mathrm{H}^{\mathrm{V}}=\mathrm{RT}^{2} \Delta \mathrm{Z}(\mathrm{d} \ln \mathrm{P} / \mathrm{dT})$

7). Surface tension, $\sigma=0.1386\left(1-\mathrm{T}_{\mathrm{r}}\right)^{1.6866},(\mathrm{~N} / \mathrm{m})$

8) Vis cosity, vs: $\log$ vs $(\mathrm{cP})=656.25(1 / \mathrm{T}-1 / 238.16)$ 
APPENDIX A (continued)

A5 - m- Cresol, $\mathrm{C}_{7} \mathrm{H}_{8} \mathrm{O}$

1) Molecular Weight, M

108.14

2) $\mathrm{T}_{\mathrm{c}}(\mathrm{K}), \mathrm{P}_{\mathrm{c}}(\mathrm{atm}), \mathrm{V}_{\mathrm{c}}\left(\mathrm{cm}^{3} / \mathrm{gmol}\right), \mathrm{Z}_{\mathrm{c}}$

$705.8,45.0,310.0,0.248$

3) Acentric factor; dipole moment

$\dot{\omega}=0.464, \mu=1.80 \mathrm{D}$

4) $T_{t p}(K), T_{b}(K)$

$284.1,475.4$

5) At $293.16 \mathrm{~K}, \rho_{1}\left(\mathrm{~g} / \mathrm{cm}^{3}\right)$

1.034

6) At $298.16 \mathrm{~K}$

$\mathrm{Cp}_{\mathrm{p}}(1)$

$\mathrm{Cp}^{\circ}$

$\mathrm{S}^{0} \quad \Delta \mathrm{H}^{\mathrm{V}}$

$\Delta \mathrm{H}_{\mathrm{f}}{ }^{\mathrm{O}}$

$\Delta G_{f}{ }^{0}$

a)

55.29 (cals /gmol, K)

85.27

11.33

$3 \begin{array}{lll}-31.63 & -9.69\end{array}$

b)

$$
231.4 \underset{(k J / k g m o l,}{122.5})^{356.9}
$$
$47,427 \quad-132,403-40,562$
(kJ/kgmol)

\section{Equations}

1) Liquid volume, $v_{1}=15.0581\left(5.7+3 \mathrm{~T}_{\mathrm{r}}\right),\left(\mathrm{cm}^{3} / \mathrm{gmol}\right)$

2) Vapor pressure: In $\mathrm{P}($ torr $)=18.3036-3816.44 /(\mathrm{T}-46.13)$

$$
\ln \mathrm{P}(\mathrm{atm})=7.66037-1479.07 / \mathrm{T}-1030280 / \mathrm{T}^{2}
$$

3) Mole $\mathrm{CP}_{\mathrm{p}}{ }^{\circ} / \mathrm{R}=-0.366755+0.0587816 \mathrm{~T}-2.42517 \mathrm{E}-5 \mathrm{~T}^{2}$

4) Mole $\mathrm{C}_{\mathrm{p}}(1)=559.336-1.86259 \mathrm{~T}+0.2258292 \mathrm{E}-2 \mathrm{~T}^{2},(\mathrm{~kJ} / \mathrm{kgmol}, \mathrm{K})$ (ass umed $s$ ame as o - Cresol)

5) $\Delta \mathrm{H}^{\mathrm{V}}=\mathrm{RT}^{2} \Delta \mathrm{Z}(\mathrm{d} \ln \mathrm{P} / \mathrm{dT})$

6) Viscosity, vs: $\log$ vs $(\mathrm{CD})=1785.6(1 / \mathrm{T}-1 / 370.75)$ 
APPENDIX A (continued)

A6 - p - Cres ol, $\mathrm{C}_{7} \mathrm{H}_{8} \mathrm{O}$

1) Molecular Weight, $M$

108.14

2) $T_{c}(K), P_{c}(a t m), V_{c}\left(\mathrm{~cm}^{3} / g m o l\right), Z_{c}$

$704.6,50.8,318.0,0.246$

3) Acentric factor; dipole moment

$\omega=0.515, \mu=1.60 \mathrm{D}$

4) $T_{t p}(K), T_{b}(K)$

$308.7,475.1$

5) At $293.16 \mathrm{~K}, \rho_{1}\left(\mathrm{~g} / \mathrm{cm}^{3}\right)$

1.035

6) At $298.16 \mathrm{~K}$

$\mathrm{Cp}_{\mathrm{p}}(1)$

$\mathrm{Cp}^{0}$

$\mathrm{S}^{0} \quad \Delta \mathrm{H}^{\mathrm{V}}$

$\Delta \mathrm{H}_{\mathrm{f}}^{\mathrm{O}} \quad \Delta \mathrm{G}_{\mathrm{f}}{ }^{\mathrm{O}}$

a)

55.29

29.75

83.09

11.34

(cals /gmol, K)

$-29.97 \quad-7.38$

b)

$231.4 \quad 124.5 \quad 347.8$

(kJ/kgmol, K)

$47,469-125,454-30,893$

(kJ/kgmol)

\section{Equations}

1) Liquid volume, $\mathrm{v}_{1}=15.0901\left(5.7+3 \mathrm{~T}_{\mathrm{r}}\right),\left(\mathrm{cm}^{3} / \mathrm{gmol}\right)$

2) Vajor pressure: In $\mathrm{P}($ torr $)=16.1989-3479.39 /(\mathrm{T}-111.3)$

$$
\ln P(a t m)=8.90052-2416.26 / T-861641 / T^{2}
$$

3) Mole $\mathrm{CP}_{\mathrm{p}}^{0} / \mathrm{R}=0.105993+0.0572001 \mathrm{~T}-2.31614 \mathrm{E}-5 \mathrm{~T}^{2}$

4) Mole $\mathrm{C}_{\mathrm{p}}(1)=559.336-1.36259 \mathrm{~T}+0.2258292 \mathrm{E}-2 \mathrm{~T}^{2},(\mathrm{~kJ} / \mathrm{kgmol}, \mathrm{K})$ (ass umed s ame as o - Cresol)

5) $\Delta \mathrm{H}^{\mathrm{V}}=\mathrm{RT}^{2} \Delta \mathrm{Z}(\mathrm{d} \ln \mathrm{P} / \mathrm{dT})$

6) Viscosity, vs: $\log$ vs $(\mathrm{cp})=1826.9(1 / \mathrm{T}-1 / 372.68)$ 
APPENDIX A (continued)

A 7 - 2,3 - Xy lenol, $\mathrm{C}_{8} \mathrm{H}_{10} \mathrm{O}$

1) Molecular Weigh $t, M$

122.17

2) $\mathrm{T}_{\mathrm{c}}(\mathrm{K}), \mathrm{P}_{\mathrm{c}}(\mathrm{atm}), \mathrm{V}_{\mathrm{c}}\left(\mathrm{cm}^{3} / \mathrm{gmol}\right), \mathrm{Z}_{\mathrm{c}}$

$722.9,48.0,310.0,0.251$

3) Acentric factor; dipole moment

$\omega=0.464, \mu=1.80 \mathrm{D}$

4) $\mathrm{T}_{\mathrm{tp}}(\mathrm{K}), \mathrm{T}_{\mathrm{b}}(\mathrm{K})$

$348.2,491.2$

5) At $298.16 \mathrm{~K}, \rho_{1}\left(\mathrm{~g} / \mathrm{cm}^{3}\right)$

1.1695

6) At $298.16 \mathrm{~K}$

$C_{P}(1)$

$\mathrm{Cp}^{\circ}$

$\mathrm{S}^{\circ} \quad \Delta \mathrm{H}^{\mathrm{V}}$

$\Delta \mathrm{H}_{\mathrm{f}}{ }^{\mathrm{O}}$

$\Delta G_{\mathrm{f}}{ }^{\circ}$

a)

$62.34 \begin{gathered}25.66 \\ (\text { cals /gmol, K) }\end{gathered}$

93.51

11.33

$3 \begin{array}{cc}-37.57 & -9.69\end{array}$

b)

$261.0 \underset{(\mathrm{kJ} / \mathrm{kgmol}, \mathrm{K})}{107.4} 391.4$

$47,427-157,268-40,562$

(kJ/kgmol)

\section{Equations}

1) Liquid volume, $\mathrm{v}_{1}=15.0581\left(5.7+3 \mathrm{~T}_{\mathrm{r}}\right),\left(\mathrm{cm}^{3} / \mathrm{gmol}\right)$

2) Vapor pressure: In $\mathrm{P}($ torr $)=17.2878-4274.42 /(\mathrm{T}-74.09)$

$\ln \mathrm{P}(\mathrm{atm})=9.53837-3239.93 / \mathrm{T}-657234 / \mathrm{T}^{2}$

3) Mole $\mathrm{CP}_{\mathrm{P}} \% \mathrm{R}=5.40946+8.73061 \mathrm{E}-2 \mathrm{~T}-7.24616 \mathrm{E}-5 \mathrm{~T}^{2}+2.49691 \mathrm{E}-8 \mathrm{~T}^{3}$

4) Mole $C_{p}(1)=145.723+0.386692 \mathrm{~T},(\mathrm{~kJ} / \mathrm{kgmol}, \mathrm{K})$

5) $\Delta \mathrm{H}^{\mathrm{V}}=\mathrm{RT}^{2} \Delta \mathrm{Z}(\mathrm{d} \ln \mathrm{P} / \mathrm{dT})$

6) Vis cosity, vs: $\log$ vs $(\mathrm{cp})=1785.6(1 / \mathrm{T}-1 / 370.75)$ 
APPENDIX A (continued)

A 8 - Hydrogen, $\mathrm{H}_{2}$

1) Molecular Weight, M 2.016

2) $\mathrm{T}_{\mathrm{c}}(\mathrm{K}), \mathrm{P}_{\mathrm{c}}(\mathrm{atm}), \mathrm{V}_{\mathrm{c}}\left(\mathrm{cm}^{3} / \mathrm{gmol}\right), \mathrm{Z}_{\mathrm{c}} \quad 33.27,12.79,65.001,0.292$

3) Acentric factor; dipole moment $\quad \omega=-0.22, \mu=0.0 \mathrm{D}$

4) $\mathrm{T}_{\mathrm{tp}}(\mathrm{K}), \mathrm{T}_{\mathrm{b}}(\mathrm{K}) \quad 14.06,20.40$

5) At $20.46 \mathrm{~K}, \rho_{1}\left(\mathrm{~g} / \mathrm{cm}^{3}\right) \quad 0.0709$

6) At $298.16 \mathrm{~K} \quad \mathrm{Cp}^{0} \quad \mathrm{~S}^{0} \quad \Delta \mathrm{H}^{\mathrm{V}} \quad \Delta \mathrm{H}_{\mathrm{f}}{ }^{0} \quad \Delta \mathrm{G}_{\mathrm{f}}{ }^{0}$

a)

$6.89 \quad 31.21$

(cals/gmol, K)

0.216

(kcal/gmol)

b)

$$
\begin{aligned}
& 28.85 \\
& (\mathrm{~kJ} / \mathrm{kgmol}, \mathrm{K})
\end{aligned}
$$

903.74

0
$(\mathrm{~kJ} / \mathrm{kgmol})$

\section{Equations}

1) Liquid volume, $\mathrm{v}_{1}=0.955\left(5.7+3 \mathrm{~T}_{\mathrm{r}}\right),\left(\mathrm{cm}^{3} / \mathrm{gmol}\right)$

2) Liquid density, $\rho_{1}=1.601406+1.1026\left[1+(1-\mathrm{T} / 33.25)^{0.272}\right]$, $\left(\mathrm{kgmo} 1 / \mathrm{m}^{3}\right)$

3) Vapor pressure: In $\mathrm{P}($ torr $)=14.7996-232.321 /(\mathrm{T}-8.08)$

$$
\ln \mathrm{P}(\mathrm{atm})=2.06561+82.9148 / \mathrm{T}-2604.56 / \mathrm{T}^{2}
$$

4) Mole $\mathrm{Cp}^{0} / \mathrm{R}=3.44471+8.38932 \mathrm{E}-5 \mathrm{~T}+9.16277 \mathrm{E}-8 \mathrm{~T}^{2}$

5) $\Delta \mathrm{H}^{\mathrm{V}}=\mathrm{RT}^{2} \Delta \mathrm{Z}(\mathrm{d} \ln \mathrm{P} / \mathrm{dT})$

6) Surface tension, $\sigma=0.5363 \mathrm{E}-2\left(1-\mathrm{T}_{\mathrm{r}}\right)^{1.074},(\mathrm{~N} / \mathrm{m})$

7) Viscosity, vs: $\log$ vs $(c p)=13.82(1 / \mathrm{T}-1 / 5.39)$ 


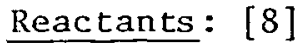

Substance

Bas is

Price

Phenol

synthetic, tank cars

$\$ 0.46 / 1 b$

Methanol

synthetic, barges

$0.49 / \mathrm{gal}$

$0.074 / 1 b$

Benzene

industrial, barges

$1.15 /$ gal

$0.156 / 1 b$

n hexane

industrial, tank cars

$0.74 / \mathrm{gaI}$

$0.134 / 1 b$

$95 \%$, tank cars

$0.85 / \mathrm{gal}$

$0.154 / 1 b$

n heptane

industrial, tank cars

$0.68 / \mathrm{ga} 1$

$0.179 / 1 b$

95\%, tank cars

$0.95 / \mathrm{gaI}$

$0.166 / 1 b$

Products: [8]

Subs tance

Bas is

Price

o-Cresol

99\% pure, bulk

$\$ 0.58 / 1 b$

m-Cresol

$95-98 \%$, drums

$1.71 / 1 b$

tanks

$1.65 / 1 b$

$p$ - Cres ol

bulk

$1.30 / 1 b$

$\mathrm{m}, \mathrm{p}-$ Cresol

bulk

$0.82 / 1 b$

mixed xylenols

bulk

$0.58 / 1 b$

Gases (current Gulf Coast prices):

$\begin{array}{lccc}\text { Substance } & \mathrm{Mi} & \$ / 10^{6} \mathrm{Btu} & \$ / 1 \mathrm{~b} \\ \text { Hydrogen }\left(\mathrm{H}_{2}\right) & 2.016 & 1.30 & 0.0792 \\ \text { Methane }\left(\mathrm{CH}_{4}\right) & 16.042 & 1.30 & 0.0310\end{array}$


APPENDIX C: PLANT UTILITIES \& COST

(Operating hours/y: Reaction Section $=2500 ;$ Separation Section $=8400)$

$\underline{\text { Reaction Section }}$

\begin{tabular}{|c|c|c|c|c|}
\hline Utility & Amount & Unit Cost & $\$ / \mathrm{h}$ & $\$ / y$ \\
\hline
\end{tabular}

$\begin{array}{lllll}1) \mathrm{CW} & 2.103 \mathrm{E} 4 \mathrm{lbm} / \mathrm{h} & \$ 0.10 / 1000 \mathrm{gal} & 0.2519 & 6.298 \mathrm{E} 2 \\ & 2.519 \mathrm{E} 3 \mathrm{gal} / \mathrm{h}\end{array}$

2) Steam, 500 psia $\quad 1.784 \mathrm{E} 31 \mathrm{bm} / \mathrm{h} \quad \$ 2.20 / 10001 \mathrm{bm} \quad 3.925 \quad 9.812 \mathrm{E} 3$

3) Electrical power, *
a) pumps
$1.587 \mathrm{E} 4 \mathrm{~kJ} / \mathrm{h}$
$\$ 0.041 / \mathrm{klVh}$
$0.1807 \quad 4.519 \mathrm{E} 2$
b) lights, instru., comp.
$1.587 \mathrm{E} 4 \mathrm{~kJ} / \mathrm{h}$
$\$ 0.041 / \mathrm{kWh}$
0.1807
4.519E2

(for $2500 \mathrm{~h}$ )

$\$ 4.538 / \mathrm{h} \quad \$ 11,345 / \mathrm{y}$

Separation Section

\begin{tabular}{|c|c|c|c|c|c|}
\hline & Utility & Amount & Unit Cost & $\$ / \mathrm{h}$ & $\$ / y$ \\
\hline 1) & $\mathrm{CW}$ & $\begin{array}{l}9.197 \mathrm{E} 4 \mathrm{lbm} / \mathrm{h} \\
1.102 \mathrm{E} 4 \mathrm{gal} / \mathrm{h}\end{array}$ & $\$ 0.10 / 1000 \mathrm{gal}$ & 1.102 & $9.257 \mathrm{E} 3$ \\
\hline 2) & Steam, 500 psia & $3.751 \mathrm{E} 31 \mathrm{bm} / \mathrm{h}$ & $\$ 2.20 / 10001 \mathrm{bm}$ & 8.252 & $6.932 \mathrm{E} 4$ \\
\hline 3) & $\begin{array}{l}\text { Electrical power, * } \\
\text { a) pumps }\end{array}$ & $6.953 \mathrm{E} 3 \mathrm{~kJ} / \mathrm{h}$ & $\$ 0.041 / \mathrm{kWh}$ & 0.07919 & $6.652 \mathrm{E} 2$ \\
\hline & $\begin{array}{l}\text { b) lights, instru., } \\
\text { comp. }\end{array}$ & $6.953 \mathrm{E} 3 \mathrm{~kJ} / \mathrm{h}$ & $\$ 0.041 / \mathrm{kiVh}$ & 0.07919 & $6.652 \mathrm{E} 2$ \\
\hline
\end{tabular}

(for $8400 \mathrm{~h}$ )

$\$ 9.512 / \mathrm{h} \quad \$ 79,907 / y$ 

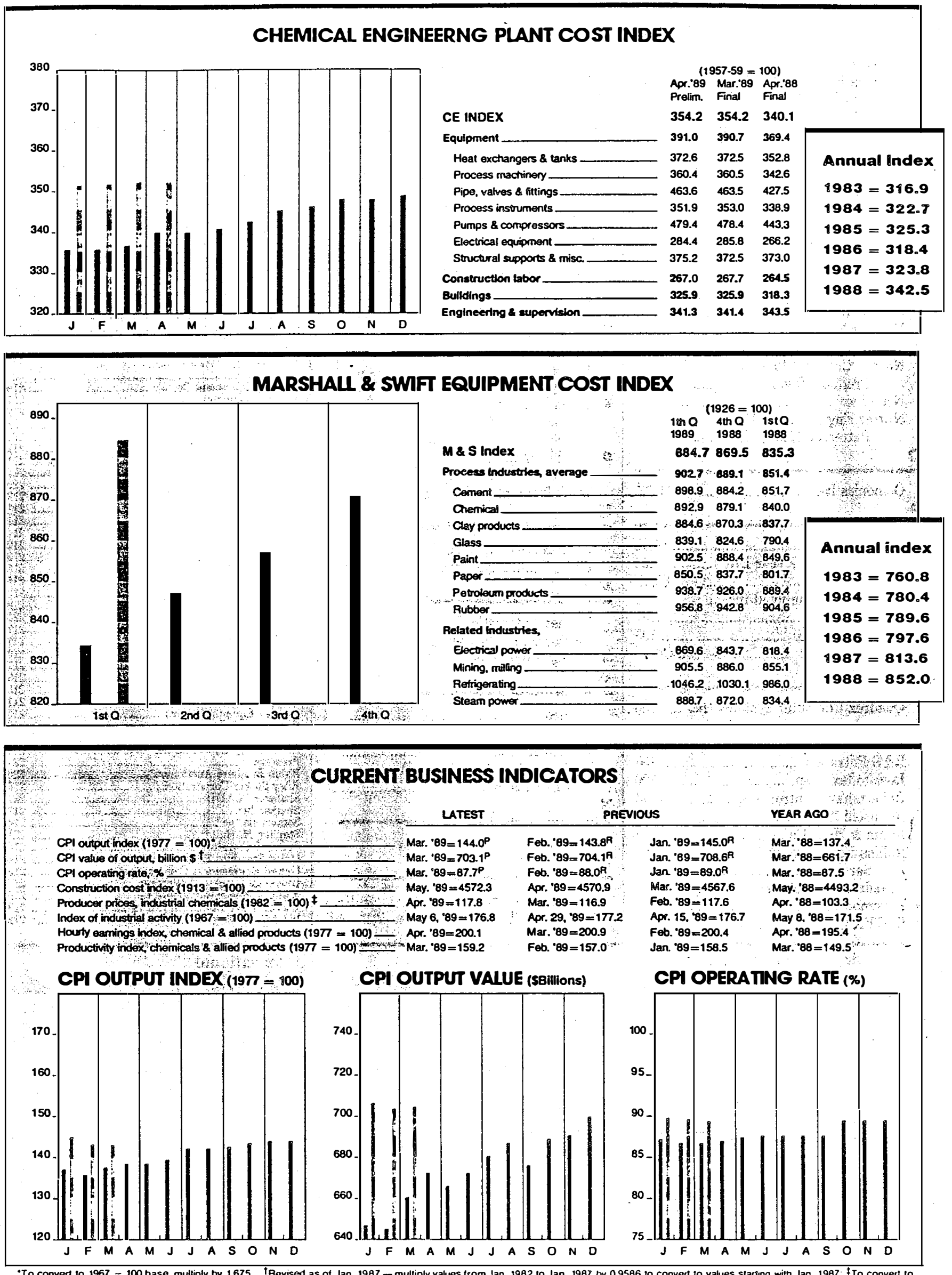

To convert to 1967 = 100 base. multiply by 1.675 . Tfevised as of Jan. 1987 - multiply values trom Jan 1982 to Jan. 1987 by 0.9586 to convent to values starting with Jan. 1987 : 7 To convert to $1967=100$ base. multiply by 3.524: 5 To convert to $1967=100$ base. multiply by 3.5241. P = Pretiminary: $A=$ Revised. For an explanation and additional intormation calt: (212) 512.6931 or (212) 512.6793 
nupper case (chemical element symbols not included)

A

c, c。

$\mathrm{C}_{\mathbf{2}}(\mathbf{1}), \mathrm{C}_{\mathbf{2}}^{\circ}$

CX

$\mathbf{F}, \mathbf{F}$ 。

$\Delta \mathbf{G e}^{\circ}$

G, L

Gmax

$\Delta H^{*}, \Delta H^{\top}$

Ic, Inc, PI

I.K, HK

Mi

MC

ocx

P, Pe

PF

POC, Top

Q. Qsolar

Re

REAC, Rm

$\mathbf{S}^{\circ}$

sf, ss, Ts

$T_{\mathbf{b}}, \mathbf{T}_{\mathbf{e}}, \mathbf{T}_{\mathbf{t}}$

$\Delta T \mathbf{T r}$

$\mathbf{U}$

$\mathbf{Y}, \mathbf{Y}(\mathbf{I}), \mathbf{Y e}$

Ymax

$\mathbf{X i}, \mathbf{Y i}$

$\mathrm{Z}, \mathrm{Ze}$ heat transfer area

plant capital cost, correlation constant

liquid heat capacitg, perfect gas state heat capacit?

cresol-rglenol fractionator

fresh feed, base case fresh feed

Gibbs free energy of formation

gas mole flov rate, liquid mole flov rate

marimum gas flov rate

enthalpy of formation, enthalpy of raporization

cost inder, income, plant income

light key, hearg key components

molecular Feight

material of construction

o-cresol rglenol fractionator

pressure, critical pressure

primary fractionotor

plant operating cost, total operating cost

heat transfer rate, solar energy input

Regnolds number

reactor, rav material cost

perfect gas state entropy

scale factor, shell-side, tube-side

n-boiling point, critical temperature, triple point

log-mean temperature difference

orerall heat transfer coeficient

Folome, liquid rolome, critical polome

maximum rapor velocity

liquid and rapor mole fractions

compressibility factor, critical value

lover case

h

PS individwal heat transfer coefficient

liquid riscosity 


\section{Greek}

$\begin{array}{ll}\mu & \text { dipole moment } \\ \text { pe, pr } & \text { gas density, liquid density } \\ \text { o } & \text { surface tension } \\ \text { o } & \text { acentric factor }\end{array}$

-22- 


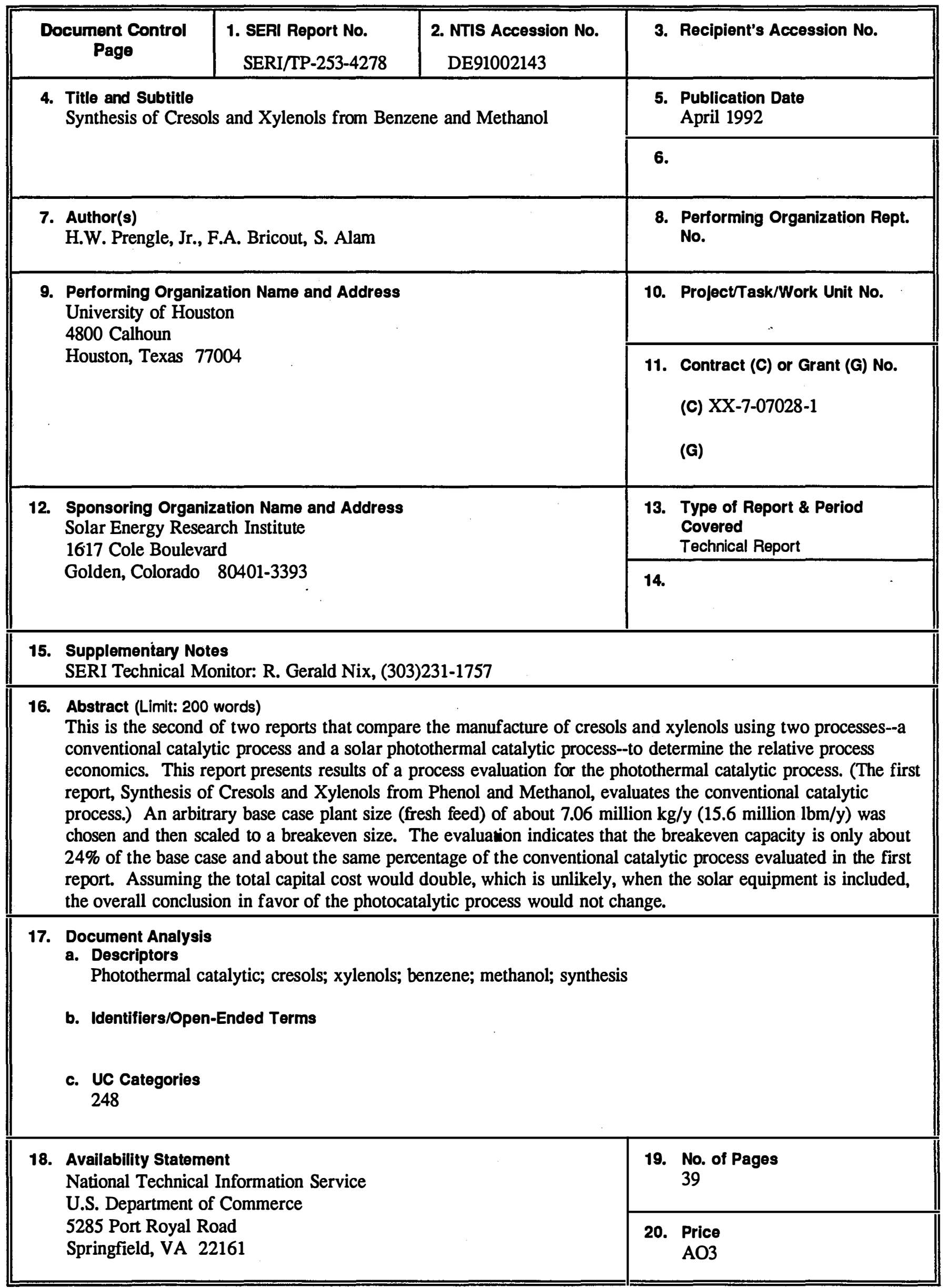

